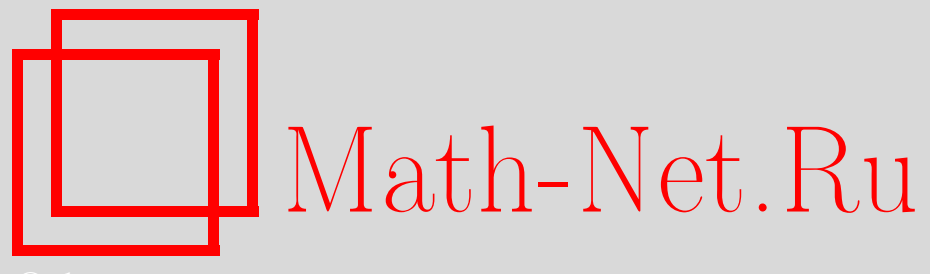

А. И. Ло, В. В. Сазонов, О состоятельности байесовских процедур, Теория вероятн. и ее примен., 2002, том 47, выпуск 2, 229-254

DOI: https://doi.org/10.4213/tvp3616

Использование Общероссийского математического портала MathNet.Ru подразумевает, что вы прочитали и согласны с пользовательским соглашением

http://www . mathnet.ru/rus/agreement

Параметры загрузки:

IP : 54.81 .137 .203

26 апреля 2023 г., 18:06:00

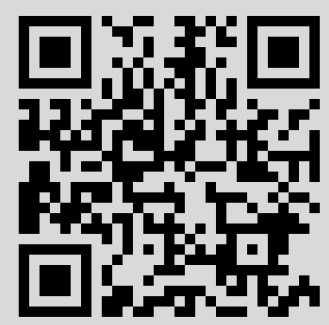




\title{
О СОСТОЯТЕЛЬНОСТИ БАЙЕСОВСКИХ ПРОЦЕДУР ${ }^{1)}$
}

\begin{abstract}
Даются достаточные условия состоятельности байесовских процедур при почти всех значениях параметра для широкого класса статистических моделей с $\sigma$-конечными априорными мерами. Для более узкого класса инвариантных статистических моделей с некоторыми $\sigma$-конечными априорными мерами приводятся условия состоятельности байесовских процедур при всех значениях параметра. Частными случаями этих инвариантных статистических моделей являются семейство сдвигов распределения на локально компактной польской группе, инвариантное относительно вращений семейство распределений на единичной сфере в $\mathbf{R}^{k}$ и семейство сдвигов и преобразований масштаба распределения в $\mathbf{R}^{k}$.
\end{abstract}

Ключевые слова и фразы: состоятельность, байесовские оценки, инвариантные марковские ядра, апостериорные распределения, инвариантные вероятностные, статистические модели.

1. Введение. Проблема состоятельности байесовских процедур является одной из центральных в байесовской статистике. Грубо говоря, состоятельность означает, что если статистик производит достаточно много наблюдений, то он (или она) в конце концов идентифицирует, какому распределению эти наблюдения подчиняются. Точнее, пусть $X_{1}, X_{2}, \ldots$ - последовательность наблюдений со значениями в некотором пространстве $\mathscr{X}$ и с совместным распределением $P_{\theta}$, зависящим от параметра $\theta$ и имеющим априорное распределение $\rho$ на параметрическом топологическом пространстве $\Theta$. Тогда апостериорные распределения $\rho^{(n)}$ параметра $\vartheta$ при заданных $X_{1}, \ldots, X_{n}$, называются состоятельными в точке $\theta$ из $\Theta$ (или, иначе, пара $\left(\theta, \rho^{(n)}\right)$ называется состоятельной), если при $n \rightarrow \infty$ распределения $\rho^{(n)}$ слабо сходится к распределению $\delta_{\theta}$, сосредоточенному в $\theta$ при $P_{\theta}$-почти всех $\theta$. Многие исследователи занимались изучением этой проблемы: Дуб [5], Ле Кам [16], Фридман [10], [11], Бреиман, Ле Кам и Шварц [1], Ло [20], Диаконис и Фридман - в серии работ,

\footnotetext{
*Hong Kong University of Science and Technology, Hong Kong; e-mail: imaylo@ust.hk

** Математический институт им. В. А. Стеклова РАН, ул. Губкина, 8, 117966 Москва, ГСП-1, Россия; e-mail: sazonov@mi.ras.ru

1) Работа выполнена при поддержке грантов RGC HKUST6189/98E для первого автора и DAG 97/98.BM60, HKUST; РФФИ 01-01-00918 для второго автора.
} 
включая замечательный обзор [4] с обсуждениями (приведенный список, конечно, далеко не исчерпывающий), однако проблема пока далека еще от ее полного решения.

Прежде, чем формулировать основные новые результаты настоящей работы, напомним некоторые известные факты о байесовской состоятельности.

Пусть $\Theta$ и $\mathscr{X}$ - борелевские подмножества польского (т.е. полного сепарабельного метрического) пространства и $P_{\theta}=\left(P_{\theta}^{\prime}\right)^{\infty}$, т.е. наблюдения независимы и одинаково распределены (н.о.р.). Тогда для гладких конечномерных семейств $P_{\theta}^{\prime}, \theta \in \Theta$, пара $\left(\theta, \rho^{(n)}\right)$ состоятельна тогда и только тогда, когда $\theta$ принадлежит носителю априорной меры $\rho$ (см. [4], а также дальнейшие содержащиеся там ссылки; для краткости и удобства мы и далее будем иногда ссылаться на работу [4] вместо оригинальных источников). С другой стороны, если семейство $P_{\theta}^{\prime}$, $\theta \in \Theta$, бесконечномерно, например, если $\mathscr{X}=\{1,2, \ldots\}$ с дискретной топологией, $\Theta$ - множество всех вероятностных мер на $\mathscr{X}$ с топологией слабой сходимости, П - пространство всех вероятностных мер с топологией Прохорова, то, как было установлено Фридманом (см., например, [4]), множество всех пар $(\theta, \rho), \theta \in \Theta, \rho \in \Pi$, для которых пары $\left(\theta, \rho^{(n)}\right)$ состоятельны, является «маленьким» множеством, а именно множеством первой категории в $\Theta \times \Pi$.

При исследовании байесовской состоятельности особое внимание уделялось задаче существования состоятельных априорных распределений, т.е. априорных распределений состоятельных при всех значениях параметра. Было показано, что популярные в байесовской статистике распределения Дирихле состоятельны, равно как и их смеси при условии, что массы параметрических мер смешиваемых распределений ограниченны. Ряд других важных априорных распределений, такие как «свободные от хвостов» и «нейтральные справа» тоже являются состоятельными (см. [4]).

Важный результат общего характера был получен Дубом в [5]: используя мартингальный подход, он показал, что идентифицируемость вместе с естественными условиями измеримости влекут состоятельность $\left(\theta, \rho^{(n)}\right)$ для любого априорного распределения $\rho$ при $\rho$-почти всех $\theta$.

В настоящей статье мы изучаем байесовскую состоятельность в общей постановке, когда параметрическое пространство $\Theta$ есть борелевское подмножество польского пространства (в случае неконечной априорной меры пространство $\Theta$ предполагается также локально компактным), пространство наблюдений $(\mathscr{X}, \mathscr{A})$ есть произвольное измеримое пространство и семейство вероятностей $P_{\theta}(A)=P\left\{\left(X_{1}, X_{2}, \ldots\right) \in\right.$ $A \mid \vartheta=\theta\}$ состоит из марковских ядер на $\Theta \times \mathscr{A}^{\infty}$, когда $\Theta$ наделено борелевской $\sigma$-алгеброй. Мы допускаем в качестве априорных мер меры необязательно конечные с тем, чтобы включить в рассмотрение некото- 
рые из таких мер, часто встречающиеся в прикладной байесовской статистике. Когда $\rho$ не является конечной, мы продолжаем по-прежнему использовать выражения типа «апостериорное распределение $\vartheta$ при заданных $X_{1}, \ldots, X_{n} \gg$ для наглядности и удобства. Точный смысл такому выражению легко придается в терминах дезынтеграции мер (см. ниже). В общем случае, если специально не оговорено противное, мы не предполагаем, что рассматриваемые наблюдения независимы по мере $P_{\theta}$ при фиксированном $\theta$.

Сначала, в разделе 2, мы показываем, что при выполнении естественных условий измеримости апостериорные распределения параметра $\theta$ при заданных значениях первых $n$ наблюдений, когда число наблюдений растет, слабо сходятся с $P_{\theta}$-вероятностью $1 \mathrm{k}$ распределению $\delta_{\theta}$, сосредоточенному в $\theta$ при почти всех (п.в.) $\theta$ из $\Theta$ относительно любой радоновской (т.е. конечной на компактных множествах) априорной меры $\rho$ на $\Theta$, или, иными словами, что байесовская процедура состоятельна при п.в. значениях параметра. Этот результат, вместе с нашим замечанием 2.2, расширяет и обобшает упомянутый выше результат Дуба [5]. Мы полагаем, что наше изложение обобщенной теоремы Дуба делает его подход более прозрачным. Подобные, но несколько иные, обобщения результатов из [5] осуществлялись также Шварцем в разделе 3 его работы [25].

Наши основные результаты содержатся в разделах 3 и 4 , где мы изучаем более сильное свойство байесовской состоятельности при всех значениях параметра предполагая дополнительно, что рассматриваемая модель обладает некоторой структурой инвариантности. Именно, мы ограничиваемся рассмотрением «инвариантной статистической модели» (cp. [8], [19]), когда имеется группа $G$ преобразований действующих на выборочном пространстве $(\mathscr{X}, \mathscr{A})$ такая, что для любого $g$ из $G$ и любого $P_{\theta}$ из рассматриваемого семейства распределений $\left\{P_{\theta}: \theta \in \Theta\right\}$ на $\left(\mathscr{X}^{\infty}, \mathscr{A}^{\infty}\right)$ сушествует единственное $\theta$ из $\Theta$, для которого $P_{\theta} g^{-1}=P_{\bar{\theta}}$ (при этом действие $g$ на элемент $x=\left(x_{1}, x_{2}, \ldots\right)$ из $\mathscr{X}^{\infty}$ понимается покоординатно: $\left.g x=\left(g x_{1}, g x_{2}, \ldots\right)\right)$. В этом случае множество отображений $\theta \rightarrow \bar{\theta}$ с композицией в качестве умножения есть группа, обозначим ее $G$, действующая на $\Theta$. Состоятельность рассматривается по отношению к априорной мере, имеющей ограниченную непрерывную положительную плотность относительно $G$-инвариантной $\sigma$-конечной меры $\pi$ на $\Theta$. При изучении общего случая не предполагается, что семейство $\left\{P_{\theta}: \theta \in \Theta\right\}$ доминированно. Частные случаи этой модели исследовались ранее Ло [20] (модель параметра сдвига: выборочное пространство и $\Theta$ есть действительная прямая $\mathbf{R}, P_{\theta}(\cdot)=P(\cdot-\theta)$ и $G$ состоит из сдвигов $\mathbf{R}$ ) и Ло совместно с Кабрерой [21] (выборочное пространство и $\Theta$ есть единичная сфера в $\mathbf{R}^{k}, G$ - группа $O_{k}$ ортогональных преобразований $\mathbf{R}^{k}$, семейство $\left\{P_{\theta}: \theta \in \Theta\right\}$ таково, что $P_{\theta}(\cdot)=P_{g \theta}(g \cdot)$ для 
всех $\theta$ и $g$ ). Показывается, что при выполнении весьма широких условий, если инвариантные версии марковских ядер, представляющих собой апостериорные распределения, существуют, байесовская процедура для инвариантной статистической модели состоятельна при всех значениях параметра. Раздел 4 посвящен собственно существованию инвариантных марковских ядер.

Частные случаи и примеры рассматриваются в разделе 5. Там показывается, в частности, что теорема 3.1 справедлива для общей модели параметра сдвига, в которой выборочное пространство $\mathscr{X}$ есть произвольная локально компактная польская группа, $G$ - группа левых сдвигов в $\mathscr{X}$, наблюдения независимы и одинаково распределены или, более общим образом, подчиняются, в некотором смысле, усиленному закону больших чисел, в предположении, что мера $P$ обладает некоторой гладкостью. Устанавливается также, что для модели параметров сдвига и масштаба на прямой (а при некоторых дополнительных ограничениях и в $\mathbf{R}^{k}$ ) с н.о.р. наблюдениями имеющими абсолютно непрерывное распределение с конечным математическим ожиданием тоже имеет место состоятельность байесовской процедуры при всех значениях параметра.

Доказательство теоремы 2.1 основано на применении мартингального подхода Дуба. Далее, при доказательстве состоятельности при всех значениях параметра в теореме 3.1 мы используем инвариантность апостериорных распределений. Вопрос существования инвариантных апостериорных распределений является весьма деликатным и далеко не простым (по крайней мере в случае недоминированного семейства распределений). На наше исследование этого вопроса большое влияние оказали идеи Л. Ле Кама из его работ [17], [18].

\section{2. Состоятельность для почти всех значений параметра.} Пусть $(\mathscr{X}, \mathscr{A})$ - измеримое пространство ( $\mathscr{A}$ есть $\sigma$-алгебра подмножеств $\mathscr{X})$ и $\left(\mathscr{X}^{m}, \mathscr{A}^{m}\right), m=1, \ldots, \infty,-$ произведение пространства $(\mathscr{X}, \mathscr{A}) m$ раз на себя. Рассмотрим последовательность $\mathscr{X}$-значных случайных величин $X_{1}, X_{2}, \ldots$ (т.е. измеримых отображений основного вероятностного пространства в $\left.\left(\mathscr{X}^{\infty}, \mathscr{A}^{\infty}\right)\right)$, представляющих наши наблюдения, и будем считать, что их совместное распределение принадлежит семейству $\left\{P_{\theta}: \theta \in \Theta\right\}$ распределений на $\left(\mathscr{X}^{\infty}, \mathscr{A}^{\infty}\right)$. Здесь $\theta-$ параметр и $\Theta$ - пространство возможных значений параметра. Пусть $\mathscr{B}-\sigma$-алгебра подмножеств $\Theta$ и $\rho-\sigma$-конечная мера на $(\Theta, \mathscr{B})$. Предположим, что $P_{\theta}^{\prime}(A)$, как функция от $\theta, \mathscr{B}$-измерима для любого фиксированного $A \in \mathscr{A}^{\infty}$ (т.е. $P .(\cdot)$ есть марковское ядро на $\left.(\Theta, \mathscr{B}) \times \mathscr{A}^{\infty}\right)$. Тогда на $\left(\mathscr{X}^{m} \times \Theta, \mathscr{A}^{m} \times \mathscr{B}\right), m=1, \ldots, \infty$, существует единственная $\sigma$-конечная мера, обозначим ее $\mu_{m}$, такая, что

$$
\mu_{m}(A \times B)=\int_{B} P_{\theta}^{(m)}(A) \rho(d \theta), \quad A \in \mathscr{A}^{m}, \quad B \in \mathscr{B}
$$


где $P_{\theta}^{(m)}(A)=P_{\theta}(\widetilde{A}), m<\infty, P_{\theta}^{(\infty)}(\cdot)=P_{\theta}(\cdot)$, и $\widetilde{A}$ есть цилиндр в $\mathscr{X}^{\infty}$ с основанием $A$. Это легко следует, например, из предложения 3.2 .1 в [22]. Обозначим $Q_{m}$ маргинальную меру, индуцированную мерой $\mu_{m}$ на $\mathscr{X}^{m}: Q_{m}(A)=\mu_{m}(A \times \Theta)$ для $A$ из $\mathscr{X}^{m}$. Легко видеть, что если $m_{1} \leqslant m_{2}$, то $\mu_{m_{1}}$ есть проекция $\mu_{m_{2}}$ и, следовательно, $Q_{m_{1}}$ есть проекция $Q_{m_{2}}$.

Лемма 2.1. Если мера $Q_{m} \sigma$-конечна (конечна) для некоторого $m=m_{0}<\infty$, то она б-конечна (конечна) для всех $m \geqslant m_{0}$. Если $\Theta$ есть борелевское подмножество польского пространства, $\mathscr{B}$ - его борелевская $\sigma$-алгебра и мера $Q_{m} \sigma$-конечна, то мера $\mu_{m}$ может быть дезынтегрирована в виде

$$
\mu_{m}(d x, d \theta)=Q_{m}(d x) \rho_{x}^{(m)}(d \theta)
$$

Выражение (2.1), конечно, означает, что существуют марковские ядра $\rho_{x}^{(m)}(B)$ на $\mathscr{X}^{m} \times \mathscr{B}$ (являющиеся вероятностными мерами на $\mathscr{B}$ для любого $x \in \mathscr{X}^{m}$ и $\mathscr{A}^{m}$-измеримые для каждого $B \in \mathscr{B}$ ) такие, что для всякого $A$ из $\mathscr{A}^{m}$ и $B$ из $\mathscr{B}$

$$
\mu_{m}(A \times B)=\int_{B} P_{\theta}^{(m)}(A) \rho(d \theta)=\int_{A} \rho_{x}^{(m)}(B) Q_{m}(d x) .
$$

Марковские ядра $\rho^{(m)}(\cdot)$ могут быть интерпретированы как регулярные условные распределения $\vartheta$ при фиксированных первых $m$ наблюдениях (см. начало доказательства теоремы 2.1).

Д о к а з а т л ь с т о. Первое утверждение леммы следует из того, что, как было отмечено выше, $Q_{m}$ есть проекция $Q_{m}$, если $m_{0} \leqslant m$. Второе утверждение является, по-видимому, известным, однако мы не смогли найти соответствующей ссылки в литературе. Во всяком случае оно легко следует из леммы 1 главы 12 в [18]: достаточно разбить $\mathscr{X}^{m}$ на подмножества $\mathscr{X}_{m p}, p=1,2, \ldots$ конечной $Q_{m}$-меры, применить упомянутую лемму из [18], чтобы дезынтегрировать меры $\mu_{m p}$, индуцированные мерой $\mu_{m}$ на $\left(\mathscr{A}^{m} \times \mathscr{B}\right) \cap\left(\mathscr{X}_{m p} \times \Theta\right)$ относительно проекций мер $\mu_{m p}$ на $\mathscr{X}_{m p}$, и затем склеить вместе полученные дезынтеграции.

Напомним, что $\delta_{\theta}$ означает вероятностную меру, сосредоточенную в $\theta$, и обозначим $(x)_{m}=\left(x_{1}, \ldots, x_{m}\right)$ для $x=\left(x_{1}, x_{2}, \ldots\right) \in \mathscr{X}^{\infty}$.

Теорема 2.1. Пусть параметрическое пространство $\Theta$ есть борелевское подмножество польского пространства, $\mathscr{B}$ - его борелевская $\sigma$-алгебра в индуиированной топологии, и пусть $\rho$ есть радоновская (т.е. конечная на компактных множествах) мера на $\mathscr{B}$. Если мера $\rho$ не конечна, мы предположим также, что пространство $\Theta$ локально компактно и что мера $Q_{m} \sigma$-конечна при некотором $m=m_{0}$. Тогда, если выполнено следующее условие:

(i) проекиия $(x, \theta) \rightarrow \theta$ пространства $\left(\mathscr{X}^{\infty} \times \Theta, \mathscr{F}\right)$ на пространство $(\Theta, \mathscr{B})$ измерима, где $\mathscr{F}$ есть б-алгебра множеств вида 
$(\mathscr{A} \times \Theta) \triangle N$ c $A \in \mathscr{A}^{\infty}$ u $\mu_{\infty}(N)=0$ (по поводу этого условия см. замечания 2.1 и 2.2 ниже), то при $m \rightarrow \infty$

$$
\rho_{(x)_{m}}^{(m)} \Rightarrow \delta_{\theta} \quad P_{\theta} \text {-n.в. для } \rho \text {-почти всех } \theta \text { из } \Theta,
$$

или, иньми словами, апостериорные распределения $\vartheta$ при заданных $X_{1}=x_{1}, \ldots, X_{m}=x_{m}$ сходятся $\kappa \delta_{\theta}$ с $P_{\theta}$-вероятностью 1 для $\rho$-почти всех значений $\theta$.

Отметим, что теорема 2.1 вместе с замечанием 2.2 (см. ниже) является обобщением хорошо известного результата Дуба [5] (см. также раздел 3 в [25]).

Д о к а з а т е л ь с т в о. Рассмотрим сначала случай, когда мера $\rho$ не конечна. Отметим, что поскольку пространство $\Theta \sigma$-компактно (например, по теореме Линделефа, так как $\Theta$ обладает счетной базой и локально компактно), мера $\rho$, будучи радоновской, $\sigma$-конечна. Нашим основным пространством будет $\left(\mathscr{X}^{\infty} \times \Theta, \mathscr{A}^{\infty} \times \mathscr{B}, \mu_{\infty}\right)$. Величины $X_{m}$ понимаются как проекции $(x, \theta) \rightarrow x_{m}$, где $x=\left(x_{1}, x_{2}, \ldots\right)$, пространства $\mathscr{X}^{\infty} \times \Theta$ на выборочное пространство $\mathscr{X}$, и $\vartheta$ есть проекция $(x, \theta) \rightarrow \theta$ пространства $\mathscr{X}^{\infty} \times \Theta$ на параметрическое пространство $\Theta$. При этом марковские ядра $\rho^{(m)}(\cdot), m=1,2, \ldots, \infty$, определенные выше как дезынтеграции по отношению к проекциям на $\mathscr{X}^{m} \sigma$-конечных мер $\mu_{m}$, можно понимать как регулярные условные распределения $\vartheta$ при заданных значениях $X_{1}, \ldots, X_{m}$ относительно $\sigma$-конечной меры $\mu_{\infty}$. Действительно, пусть для $A$ из $\mathscr{A}^{m}$, как и выше, $\widetilde{A}$ обозначает цилиндр в $\mathscr{X}^{\infty}$ с основанием $A$. Мы будем использовать также обозначение $\bar{f}$ (соответственно $g^{*}$ ), где $f$ (соответственно $g$ ) есть отображение, определенное на $\Theta$ (соответственно на $\mathscr{X}^{\infty}$ ), для расширения $f$ (соответственно $g$ ) на $\mathscr{X}^{\infty} \times \Theta: \bar{f}((x, \theta))=f(\theta)$ (соответственно $\left.g^{*}((x, \theta))=g(x)\right)$. Тогда, из определения мер $Q_{m}$ и отмеченного выше факта, что при $m_{1} \leqslant m_{2}$ мера $Q_{m_{1}}$ есть проекция меры $Q_{m_{2}}$, легко получаем, что для любых $A$ из $\mathscr{A}^{m}$ и $B$ из $\mathscr{B}$

$$
\int_{\widetilde{A} \times \Theta}\left(\rho_{(\cdot)_{m}}^{(m)}(B)\right)^{*} d \mu_{\infty}=\mu_{\infty}(\widetilde{A} \times B), \quad m=m_{0}, \ldots, \infty,
$$

то есть $\rho_{(x)_{m}}^{(m)}(B)=\mathbf{E}\left(\vartheta \in B \mid X_{1}=x_{1}, \ldots, X_{m}=x_{m}\right.$ ) (здесь и ниже $\mathbf{E}$ означает математическое ожидание относительно меры $\left.\mu_{\infty}\right)$.

Далее, отметим, что для любой функции $f$ из класса $C_{0}=C_{0}(\Theta)$, действительных непрерывных функций на $\Theta$ с компактным носителем $\int|\bar{f}| d \mu_{\infty}=\int|f| d \rho<\infty$, и из (2.2) стандартным образом вытекает, что для любых $f$ из $C_{0}, A$ из $\mathscr{A}^{m}$ и $m=m_{0}, \ldots, \infty$

$$
\int_{\widetilde{A} \times \Theta}\left(\int f d \rho_{(\cdot)_{m}}^{(m)}\right)^{*} d \mu_{\infty}=\int_{\widetilde{A} \times \Theta} \bar{f} d \mu_{\infty} .
$$

Следовательно,

$$
Y_{m}(f)=\left(\int f d \rho_{(\cdot)_{m}}^{(m)}\right)^{*}, \quad m=m_{0}, \ldots, \infty
$$


- условное математическое ожидание $\bar{f}$ относительно $\sigma$-алгебры $\left(\mathscr{A}^{m}\right)^{\sim} \times \Theta$ и $Y_{m}(f), m=1, \ldots, \infty$, есть мартингал на пространстве $\left(\mathscr{X}^{\infty} \times \Theta, \mathscr{A}^{\infty} \times \mathscr{B}, \mu_{\infty}\right.$ ) (мы используем здесь и, подобным образом, ниже очевидное обозначение: $\left(\mathscr{A}^{m}\right)^{\sim} \times \Theta$ есть $\sigma$-алгебра множеств вида $\left.\widetilde{A} \times \Theta, A \in \mathscr{A}^{m}\right)$. Отсюда вытекает, что $\left\{Y_{m}(f), m=1,2, \ldots\right\}$ сходится $\mu_{\infty}$-п.в. к $\mathbf{E}\left(\bar{f} \mid \mathscr{A}^{\infty} \times \Theta\right)=Y_{\infty}(f)$ (см., например, теорему 3.1 в [2] или раздел 41.1 в [3]). Более того, поскольку пространство $C_{0}(\Theta)$ сепарабельно в равномерной норме (см., например, раздел 8.1 в [6]), существует множество $F$ в $\mathscr{A}^{\infty} \times \mathscr{B}$ такое, что $\mu_{\infty}(F)=0$ и при $(x, \theta) \notin F$

$$
Y_{m}(f)(x, \theta) \rightarrow Y_{\infty}(f)(x, \theta) \text { при всех } f \in C_{0}(\Theta) .
$$

Так как $\rho_{(x)_{m}}^{(m)}$ суть вероятностные меры, это влечет

$$
\left(\rho_{(\cdot)_{m}}^{(m)}\right)^{*} \Rightarrow\left(\rho^{(\infty)}\right)^{*} \quad \mu_{\infty} \text {-П.в. }
$$

(см., например, разделы 8.8 и 8.12 в [6]).

Далее, по условию (i) для любой функции $f$ из $C_{0}(\Theta)$

$$
\left(\int f d \rho .^{(\infty)}\right)^{*}=\mathbf{E}\left(\bar{f} \mid \mathscr{A}^{\infty} \times \Theta\right)=\mathbf{E}(\bar{f} \mid \mathscr{F})=\bar{f} \quad \mu_{\infty} \text {-п.в. }
$$

и, более того, в силу сепарабельности $C_{0}(\Theta)$ в равномерной норме $(2.3)$ справедливо на некотором множестве полной меры $\mu_{\infty}$ одном и том же для всех функций $f$ из $C_{0}(\Theta)$. Следовательно,

$$
\left(\rho_{(\cdot)_{m}}^{(m)}\right)^{*}(x, \theta) \Rightarrow\left(\rho^{(\infty)}\right)^{*}(x, \theta)=\delta_{\theta} \mu_{\infty} \text {-П.в. }
$$

Наконец, поскольку для любого множества $G$ из $\mathscr{A}^{\infty} \times \mathscr{B}$

$$
\mu_{\infty}(G)=\int P_{\theta}\left(G_{\theta}\right) \rho(d \theta)
$$

где $G_{\theta}$ - сечение $G$ в $\theta,(2.4)$ влечет

$$
P_{\theta}\left(x: \rho_{(x)_{m}}^{(m)} \Rightarrow \delta_{\theta}\right)=1 \quad \rho \text {-п.в. }
$$

В случае, когда мера $\rho$ конечна, доказательство проводится аналогично с той разницей, что вместо пространства $C_{0}(\Theta)$ рассматривается последовательность $\left\{g_{k}, k=1,2, \ldots\right\}$ действительных ограниченных непрерывных функций на $\Theta$ такая, что для любых вероятностных мер $\nu, \nu_{1}, \nu_{2}, \ldots$ на $(\Theta, \mathscr{B})$ сходимость $\nu_{n} \Rightarrow \nu$ эквивалентна сходимости $\int g_{k} d \nu_{n} \rightarrow \int g_{k} d \nu_{n}$ при всех $k=1,2, \ldots$ (доказательство существования такой последовательности можно найти, например, в [23], глава 2, теоремы $6.1,6.2)$.

3 а м е ч а н и е 2.1. $\sigma$-алгебра $\mathscr{F}$ совпадает с $\sigma$-алгеброй, порожденной множествами вида $A \times \Theta$, где $A$ принадлежит $\mathscr{A}^{\infty}$, и всеми множествами $\mu_{\infty}$-меры нуль. В условии (i) $\sigma$-алгебру $\mathscr{F}$ можно заменить на ее $\mu_{\infty}$-пополнение $\mathscr{F}^{*}$, которое может быть представлено в виде $\mathscr{F}^{*}=\left\{(A \times \Theta) \triangle M: A \in \mathscr{A}^{\infty}, M \subset N, \mu_{\infty}(N)=0\right\}$. 
3 а м е ч а н и е 2.2. В этом замечании, если явно не оговорено противное, $(\Theta, \mathscr{B})$ - некоторое измеримое пространство, $D$ - некоторое польское пространство с метрикой $d, \mathscr{B}_{D}-$ борелевская $\sigma$-алгебра в $D$ и $\rho$ - некоторая $\sigma$-конечная мера на $(\Theta, \mathscr{B})$. Все остальные используемые и не определяемые здесь обозначения те же, что были введены ранее.

Пусть $T$ - измеримое отображение $\Theta$ в $D$ и положим $\mathscr{B}_{1}=$ $T^{-1}\left(\mathscr{B}_{D}\right)$. Для каждого натурального $n \geqslant 1$ пусть $T_{n}$ - измеримое отображение (статистика) $\left(\mathscr{X}^{n}, \mathscr{A}^{n}\right)$ в $\left(D, \mathscr{B}_{D}\right)$. Покажем, что если

$$
P_{\theta}\left(x: T_{n}\left(x_{(n)}\right) \longrightarrow T(\theta)\right)=1 \text { для } \rho \text {-почти всех } \theta \in \Theta \text {, }
$$

то $\left(^{*}\right)$ проекиия $(x, \theta) \rightarrow \theta$ пространства $\left(\mathscr{X}^{\infty} \times \Theta, \mathscr{F}\right)$ на $\left(\Theta, \mathscr{B}_{1}\right)$ измерима, и, следовательно, условие (i) теоремь 2.1 вьполнено, если также $\mathscr{B} \subset \mathscr{B}_{1}$. Включение $\mathscr{B} \subset \mathscr{B}_{1}$ имеет место, в частности, когда T инъективно и измеримое пространство $(\Theta, \mathscr{B})$ есть борелевское подмножество польского пространства со своей борелевской б-алгеброй (это следует из результатов раздела 39.5 главы 3 в [15]; см. также, раздел 3 главьл І в [23]).

Действительно, обозначим $\bar{T}$ (соответственно $\widetilde{T}_{n}$ ) расширение $T$ (соответственно $T_{n}$ ) на $\mathscr{X} \infty \times \Theta: \bar{T}(x, \theta)=T(\theta)$ (соответственно $\left.\widetilde{T}_{n}(x, \theta)=T_{n}\left(x_{(n)}\right)\right)$. Ясно, что оба отображения $\bar{T}$ и $\widetilde{T}_{n}\left(\mathscr{A}^{\infty} \times \mathscr{B}\right)$ измеримы. Более того, множество, где отображения $T_{n}$ сходятся, и их предел на нем, тоже измеримы (см., например, раздел 5.3 в [6]). Следовательно,

$$
C \stackrel{\text { def }}{=}\left\{(x, \theta): \bar{T}(x, \theta)=\lim _{n} \widetilde{T}_{n}(x, \theta)\right\} \in \mathscr{A}^{\infty} \times \mathscr{B},
$$

и, в силу (2.5) $P_{\theta}\left(C_{\theta}\right)=1$ для $\rho$-почти всех $\theta$ из $\Theta$, где $C_{\theta}$ есть $\theta$-сечение множества $C$. Отсюда вытекает, что $\mu_{\infty}\left(C^{c}\right)=\int P_{\theta}\left(C_{\theta}^{c}\right) \rho(d \theta)=0$. Более того, если для подмножества $H$ пространства $D$ мы положим

$$
\begin{aligned}
& A_{1}=\left\{(x, \theta): \lim _{n} \widetilde{T}_{n}(x, \theta) \in H\right\}, \\
& B_{1}=\left\{(x, \theta): \theta \in T^{-1}(H)\right\}=\{(x, \theta): \bar{T}(x, \theta) \in H\},
\end{aligned}
$$

то, в силу определения множества $C, A_{1} \cap C=B_{1} \cap C$, и, следовательно, $\left(A_{1} \triangle B_{1}\right) \cap C=\left(A_{1} \cap C\right) \triangle\left(B_{1} \cap C\right)=\varnothing$. Для произвольного множества $B$ из $\mathscr{B}_{1}$ пусть множество $H$ из $\mathscr{B}_{D}$ таково, что $B=T^{-1}(H)$. Тогда, с этим $H, A_{1}=A \times \Theta$ для некоторого $A$ из $\mathscr{A}^{\infty}, B_{1}=\mathscr{X}^{\infty} \times B$ принадлежит $\mathscr{A}^{\infty} \times \mathscr{B}$ и, согласно отмеченному выше, $\mu_{\infty}\left(A_{1} \triangle B_{1}\right)=0$, откуда вытекает, что множество $B_{1}=A_{1} \triangle\left(A_{1} \triangle B_{1}\right)$ принадлежит $\mathscr{F}$.

Приведем один важный частный случай, когда условие (2.5) выполнено (ср. [5]). Пусть $\mathscr{X}$ - польское пространство и выберем в качестве $D$ польское пространство $\mathscr{P}$ всех борелевских вероятностных мер на $\mathscr{X}$ с метрикой Прохорова. Положим

$$
T_{n}\left(x_{(n)}\right)=\frac{1}{n} \sum_{1}^{n} \delta_{x_{i}} .
$$


Тогда, по теореме Варадарайна (см., например, раздел 11.4 в [7]), условие (2.5) выполнено с $T(\theta)=P_{\theta}^{(1)}$, где $P_{\theta}^{(1)}$ есть распределение $X_{1}$ относительно $P_{\theta}$, если наблюдения $X_{1}, X_{2}, \ldots$ независимы и одинаково распределены для $\rho$-почти всех $\theta$ из $\Theta$. В действительности из доказательства теоремы Варадарайна следует, что в рассматриваемом случае условие (2.5) выполнено, если для $\rho$-почти всех $\theta$ из $\Theta$

$$
P_{\theta}\left(x: \frac{1}{n} \sum_{1}^{n} f\left(x_{i}\right) \rightarrow T(\theta)\right)=1 \quad \text { для всех } f \in C(\mathscr{X}),
$$

где $T(\theta)$ есть некоторое $\mathscr{P}$-значное измеримое отображение и $C(\mathscr{X})-$ пространство всех действительных ограниченных непрерывных функций на $\mathscr{X}$. Конечно, $(2.7)$ является одной из форм усиленного закона больших чисел для $X_{1}, X_{2}, \ldots$. В частности, (2.7) выполняется с $T(\theta)=P_{\theta}^{(1)}$, если последовательность $X_{1}, X_{2}, \ldots$ стационарна и эргодична для $\rho$-почти всех $\theta$ из $\Theta$.

Естественно задаться вопросом: будет ли утверждение $(*)$ попрежнему справедливо, если в (2.5) сходимость $P_{\theta}$-п.в. заменить на сходимость по вероятности относительно $P_{\theta}$, т.е., если вместо $(2.5)$ выполнено условие

$$
\lim _{n} P_{\theta}\left(x: d\left(T_{n}\left(x_{(n)}\right), T(\theta)\right)>\varepsilon\right)=0 \quad \text { для } \rho \text {-почти всех } \theta \in \Theta \text { и } \varepsilon>0 .
$$

При выполнении $\left(2.5^{*}\right)$ для любого фиксированного $\varepsilon>0$ по теореме о мажорированной сходимости мы имеем $\mu_{\infty}\left((x, \theta): d\left(\widetilde{T}_{n}(x, \theta), \bar{T}(x, \theta)>\right.\right.$ $\varepsilon)=\int P_{\theta}\left(x: d\left(T_{n}\left(x_{(n)}\right), T(\theta)\right)>\varepsilon\right) \rho(d \theta) \longrightarrow 0$ при $n \rightarrow \infty$, если для некоторого натурального $n_{0}$

$$
\int \sup _{n \geqslant n_{0}} P_{\theta}\left(x: d\left(T_{n}\left(x_{(n)}\right), T(\theta)\right)>\varepsilon\right) \rho(d \theta)<\infty .
$$

Следовательно, если выполнено условие $\left(2.5^{*}\right)$ из справедливости (2.8) для всех $\varepsilon>0$ вытекает, что $\widetilde{T}_{n}$ сходится к $\bar{T}$ по мере $\mu_{\infty}$, так что существует подпоследовательность $\left\{n^{\prime}\right\}$ последовательности натуральных чисел такая, что $\widetilde{T}_{n^{\prime}} \rightarrow \bar{T} \mu_{\infty}$-П.в. Если мы определим теперь $C^{\prime}$ также, как $C$ в (2.6), но с $n^{\prime}$ вместо $n$, то мы получим $\mu_{\infty}\left(\left(C^{\prime}\right)^{c}\right)=0$, и такое же рассуждение, как и выше, показывает, что утверждение $\left({ }^{*}\right)$ справедливо. Таким образом, справедливость $\left(2.5^{*}\right)$ вместе с выполнением (2.8) при всех $\varepsilon>0$ является достаточным условием для $(*)$. Конечно, условие (2.8) автоматически выполнено, если мера $\rho$ конечна.

3. Состоятельность для всех значений параметра. При изучении байесовской состоятельности для всех значений параметра мы ограничимся рассмотрением моделей, имеющих некоторую инвариантную структуру. 
Пусть $(\mathscr{X}, \mathscr{A})$ - измеримое пространство и $G$ - группа, действующая слева на $\mathscr{X}$ (т.е. каждой паре $(g, x) \in G \times \mathscr{X}$ соответствует единственный элемент $g x \in \mathscr{X}$ так, что $g_{1}\left(g_{2} x\right)=\left(g_{1} \circ g_{2}\right) x$ и $e x=x$; см., например, раздел 6.1 в [8]). Отображения $x \rightarrow g x, g \in G$, пространства $\mathscr{X}$ в себя, как известно, биективны и мы предположим, что они измеримы. Пусть, как и выше, $\left(\mathscr{X}^{m}, \mathscr{A}^{m}\right), m=1,2, \ldots, \infty$, означает $m$-кратное произведение $(\mathscr{X}, \mathscr{A})$ на себя. Действие группы $G$ мы распространим также на $\mathscr{X}^{m}$, положив $g x=\left(g x_{1}, g x_{2}, \ldots\right)$ для $x=\left(x_{1}, x_{2}, \ldots\right)$ из $\mathscr{X}^{m}$.

Рассмотрим последовательность $X_{1}, X_{2}, \ldots \mathscr{X}$-значных случайных величин, представляющих наши наблюдения (так что $\mathscr{X}$ есть наше выборочное пространство) и предположим, что их совместное распределение принадлежит параметрическому семейству $\left\{P_{\theta}: \theta \in \Theta\right\}$ распределений на $\left(\mathscr{X}^{\infty}, \mathscr{A}^{\infty}\right)$ являюшемуся $G$-инвариантным, т.е. для любых $g$ из $G$ и $\theta$ из $\Theta$ существует единственный элемент из $\Theta$, обозначаемый $\bar{g} \theta$, такой, что

$$
P_{\theta} g^{-1}(A)=P_{\theta}\left(g^{-1} A\right)=P_{\bar{g} \theta}(A), \quad A \in \mathscr{A}^{\infty} .
$$

Описанную структуру мы называем $G$-инвариантной статистической моделью (она является частным случаем $G$-инвариантной вероятностной модели Итона, см. [8], раздел 7.3).

Напомним, что $G$-инвариантность $\left\{P_{\theta}: \theta \in \Theta\right\}$ влечет взаимную однозначность параметризации, т.е. $P_{\theta_{1}} \neq P_{\theta_{2}}$ при $\theta_{1} \neq \theta_{2}$ (это непосредственно вытекает из определения: если $\theta_{1} \neq \theta_{2}$ и $е$ есть единичный элемент в $G$, то $P_{\theta_{1}}=P_{\theta_{1}} e^{-1}$ не может совпадать с $P_{\theta_{2}}$ в силу единственности в определении $G$-инвариантности семейства $\left.\left\{P_{\theta}: \theta \in \Theta\right\}\right)$. В терминах величин $X_{1}, X_{2}, \ldots$ (3.1) означает, что если $X_{1}, X_{2}, \ldots$ имеют совместное распределение $P_{\theta}$, то совместное распределение величин $g X_{1}, g X_{2}, \ldots$ есть $P_{\bar{g} \theta}$. Как хорошо известно (см., например, предложение 7.10 в [8]), отображения $\theta \rightarrow \bar{g} \theta$ пространства $\Theta$ в себя суть биекции и с композицией в качестве умножения $\bar{G}=\{\bar{g}: g \in G\}$ есть группа, являющаяся гомоморфным образом $G$.

Относительно пространства $\Theta$ мы предположим, что оно есть борелевское подмножество польского пространства и обозначим $\mathscr{B} \sigma$-алгебру его борелевских подмножеств. Далее, мы предположим, что $P_{\theta}(A)$ есть марковское ядро на $(\Theta, \mathscr{B}) \times \mathscr{A}^{\infty}$ и что на $(\Theta, \mathscr{B})$ существует $G$-инвариантная (т.е. $\pi(\bar{g} B)=\pi(B)$ для всех $\bar{g}$ из $\bar{G}$ и $B$ из $\mathscr{B}) \sigma$-конечная мера $\pi$. Ниже $\pi$ всегда будет обозначать такую меру и, если не оговорено противное, $\pi$ будет выбрана в качестве априорной меры. Мера $\pi$ существует, например, если пространство $\Theta$ также локально компактно, а группа $G$ состоит из гомеоморфизмов и является равностепенно непрерывной и почти транзитивной, т.е. орбита каждого элемента $\theta$ плотна в $\Theta$ (см. раздел 14.3 в [24]). Отметим еще, что мера $\pi$ существует во всех примерах, рассматриваемых в разделе 5. Наконец, мы предполо- 
жим, что для любого $\bar{g}$ из $\bar{G}$ отображение $\theta \rightarrow \bar{g} \theta$ пространства $\Theta$ на себя непрерывно.

Определим $\mu_{m}, P_{\theta}^{(m)}, Q_{m}$ и $\pi_{x}^{(m)}$ как в разделе 2 и отметим, что согласно (3.1) при $m<\infty$

$$
P_{\theta}^{(m)}\left(g^{-1} A\right)=P_{\theta}\left(g^{-1} \widetilde{A}\right)=P_{\bar{g} \theta}(\widetilde{A})=P_{\bar{g} \theta}^{(m)}(A)
$$

при всех $\theta$ из $\Theta, g$ из $G$ и $A$ из $\mathscr{A}^{m}$, т.е. семейство $\left\{P_{\theta}^{(m)}: \theta \in \Theta\right\}$ тоже $G$-инвариантно.

Лемма 3.1. Мерьи $Q_{m} G$-инвариантнь $и$, если мера $Q_{m} \sigma$-конечна для некоторого $m_{0}<\infty$, то для любого $m \geqslant m_{0}$ марковские ядра $\pi^{(m)}(\cdot)$ почти $(G-\bar{G})$-инвариантны, т.е. для любых $g$ из $G$ и $B$ из $\mathscr{B}$

$$
\pi_{x}^{(m)}(B)=\pi_{g x}^{(m)}(\bar{g} B)
$$

$Q_{m}$-n.в.; множество, где (3.2) не выполняется, в общем случае может зависеть от $g$ и $B$.

3 а м е ч а н и е 3.1. Утверждение леммы 3.1 относительно $\pi^{(m)}(\cdot)$ является обобщением на марковские ядра соответствующего утверждения для плотностей, содержащегося в разделе 7.3 в [8].

3 а м е ч а н и е 3.2. Свойство $G$-инвариантности мер $\pi$ и $Q_{m}$ и свойство почти $(G-\bar{G})$-инвариантности марковских ядер $\pi .^{(m)}(\cdot)$ эквивалентно могут быть выражены (соответственно) следующим образом: для любых $\mathscr{B}$-измеримой функции $\phi \geqslant 0$ и $g$ из $G$

$$
\int \phi(\theta) \pi(d \theta)=\int \phi(\bar{g} \theta) \pi(d \theta)
$$

для любых $\mathscr{A}^{m}$-измеримой функции $f \geqslant 0$ и $g$ из $G$

$$
\int f(x) Q_{m}(d x)=\int f(g x) Q_{m}(d x) ;
$$

для любых действительной ограниченной $\mathscr{B}$-измеримой функции $h$ и $g$ из $G$

$$
\int h(\theta) \pi_{x}^{(m)}(d \theta)=\int h\left(\bar{g}^{-1} \theta\right) \pi_{g x}^{(m)}(d \theta) \quad Q_{m} \text {-п.в. }
$$

причем множество, где $\left(3.2^{*}\right)$ не выполняется, в общем случае может зависеть от $g$ и $h$.

В этом можно убедиться с помощью стандартного рассуждения: начать с неотрицательных конечнозначных измеримых функций $\phi, f$ и $h$, затем представить произвольную неотрицательную измеримую функцию как предел возрастающей последовательности неотрицательных конечнозначных измеримых функций и применить теорему БеппоЛеви; в последнем случае нужно представить также функцию $h$ в виде $\max (h, 0)-\max (-h, 0)$. 
Д ок а з а те льс т в о ле м м ы 3.1 . В силу $\left(3.1^{*}\right)$ и (3.3) для любых $g$ из $G$ и $A$ из $\mathscr{A}^{m}$ имеем

$$
\begin{aligned}
Q_{m}(g A) & =\int P_{\theta}^{(m)}(g A) \pi(d \theta)=\int P_{\bar{g}^{-1} \theta}^{(m)}(A) \pi(d \theta) \\
& =\int P_{\theta}^{(m)}(A) \pi(d \theta)=Q_{m}(A) .
\end{aligned}
$$

Далее, при $m \geqslant m_{0}$, используя $(3.4),\left(2.1^{*}\right)$ с $\rho=\pi,\left(3.1^{*}\right)$ и $(3.3)$, для любых $g$ из $G$ и $A$ из $\mathscr{A}^{m}$ и $B$ из $\mathscr{B}$

$$
\begin{aligned}
\int_{A} & \pi_{g x}^{(m)}(\bar{g} B) Q_{m}(d x)=\int 1_{g A}(x) \pi_{x}^{(m)}(\bar{g} B) Q_{m}(d x) \\
= & \int 1_{\bar{g} B}(\theta) P_{\theta}^{(m)}(g A) \pi(d \theta)=\int 1_{B}\left(\bar{g}^{-1} \theta\right) P_{\bar{g}^{-1} \theta}^{(m)}(A) \pi(d \theta) \\
= & \int 1_{B}(\theta) P_{\theta}^{(m)}(A) \pi(d \theta)=\int_{A} \pi_{x}^{(m)}(B) Q_{m}(d x) .
\end{aligned}
$$

Согласно лемме 3.1 марковские ядра $\rho .^{(m)}(\cdot)$, определенные в лемме 2.1 , почти $(G-\bar{G})$-инвариантны, однако в приводимом ниже доказательстве теоремы 3.1 нам нужно более сильное свойство $(G-\bar{G})$-инвариантности ядер $\pi^{(m)}(\cdot)$ при всех $m \geqslant m_{0}$. Возникает вопрос когда существуют $(G-\bar{G})$-инвариантные версии ядер $\pi^{(m)}(\cdot)$ ? То есть когда существуют марковские ядра $\pi^{(m)}(\cdot)$, удовлетворяющие условию $\left(2.1^{*}\right)$ с $\rho=\pi$ и условию (3.2) при всех $x$ из $\mathscr{X}^{m}, g$ из $G$ и $B$ из $\mathscr{B} ?$ Мы отложим изучение этого вопроса до раздела 4 и в теореме 3.1 будем просто предполагать, что $(G-\bar{G})$-инвариантные версии ядер $\pi^{(m)}(\cdot)$ (в несколько обобщенном смысле) существуют.

Теорема 3.1. Пусть $\Theta, \mathscr{B}, Q_{m}$ удовлетворяют тем же условиям, что в теореме 2.1, причем в качестве $\rho$ теперь берется $G$-инвариантная радоновская мера $\pi$ на $\mathscr{B}$. Предположим, что условие (i) из теоремы 2.1 выполнено вместе со следуюшими двумя условиями:

(ii) существуют $(G-\bar{G})$-инвариантные версии $\widehat{\pi}_{.}^{(m)}(\cdot)$ марковских ядер $\pi^{(m)}(\cdot)$ (в смислле предложения 4.1) при всех $m \geqslant m_{0}$;

(iii) группа $\bar{G}$ действует транзитивно на $\Theta$ (т.е. для любых $\theta_{1}$ и $\theta_{2}$ из $\Theta$ существует элемент $\bar{g}$ группь $\bar{G}$ такой, что $\left.\theta_{1}=\bar{g} \theta_{2}\right)$.

Тогда

$$
\widehat{\pi}_{(x)_{m}}^{(m)} \Rightarrow \delta_{\theta} \quad P_{\theta} \text {-n.в. для всех } \theta \text { из } \Theta,
$$

m.е. существуют версии апостериорных распределений параметра $\vartheta$ при заданньгх $X_{1}=x_{1}, \ldots, X_{m}=x_{m}$ сходяииеся $\kappa \delta_{\theta}$ c $P_{\theta}$-вероятностью 1 для всех значений $\theta$. Более того, заключение теоремы справедливо для любых априорных мер имеющих ограниченную положительную непрерывную плотность относительно $\pi$.

Д о к а з а т е л ь с т в о. По теореме 2.1, в обозначениях введенных в начале ее доказательства, имеем

$$
\left(\pi_{(\cdot)_{m}}^{(m)}\right)^{*}(x, \theta) \Longrightarrow\left(\pi^{(\infty)}\right)^{*}(x, \theta)=\delta_{\theta} \quad \mu_{\infty} \text {-П.в. }
$$


Далее, пусть $\widehat{\pi}^{(m)}(\cdot), m \geqslant m_{0}$, - марковское ядро на $\left(\mathscr{X}^{m}, \mathscr{A}_{c}^{m}\right) \times \mathscr{B}$, где $\mathscr{A}_{c}^{m}$ есть некоторая $\sigma$-алгебра, содержашая $\sigma$-алгебру $\mathscr{A}^{m}$ и содержащаяся в $Q_{m}$-пополнении $\mathscr{A}^{m}$, такое, что $\widehat{\pi}_{y}^{(m)}=\pi_{y}^{(m)}$, если $y \notin N_{m}$, $Q_{m}\left(N_{m}\right)=0$.

Полагая $L=\left(\bigcup_{m \geqslant m_{0}} \tilde{N}_{m}\right)^{c}, M=L \times \Theta$, имеем

$$
\mu_{\infty}\left(M^{c}\right)=Q_{m}\left(\bigcup_{m \geqslant m_{0}} \tilde{N}_{m}\right) \leqslant \sum_{m \geqslant m_{0}} Q_{m}\left(N_{m}\right)=0
$$

и $\left(\widehat{\pi}_{(\cdot)_{m}}^{(m)}\right)^{*}(x, \theta)=\left(\pi_{(\cdot)_{m}}^{(m)}\right)^{*}(x, \theta)$ при всех $m \geqslant m_{0}$, если $(x, \theta) \in M$, так что, в силу (3.5) дополнение к множеству $\left\{(x, \theta):\left(\widehat{\pi}_{(\cdot)_{m}}^{(m)}\right)^{*}(x, \theta) \Longrightarrow \delta_{\theta}\right\} \cap M$ имеет $\mu_{\infty}$-меру нуль. Отсюда вытекает, подобно тому как $(2.4)$ влечет $\left(2.4^{*}\right)$, что

$$
P_{\theta}\left(\left\{x: \widehat{\pi}_{(x)_{m}}^{(m)} \Longrightarrow \delta_{\theta}\right\} \cap L\right)=1 \quad \pi \text {-п.в. }
$$

Пусть теперь $\theta_{0}$ - такой элемент пространства $\Theta$, что

$$
P_{\theta_{0}}\left(\left\{x: \widehat{\pi}_{(x)_{m}}^{(m)} \Longrightarrow \delta_{\theta_{0}}\right\} \cap L\right)=1
$$

и, более того, предположим, что $\hat{\pi}^{(m)}(\cdot), m \geqslant m_{0}$, удовлетворяет условию (ii) и условие (iii) выполнено. Тогда для произвольного $\theta$ из $\Theta$ существует элемент $\bar{g}$ группы $\bar{G}$ такой, что $\theta=\bar{g} \theta_{0}$, и

$$
\begin{aligned}
& P_{\theta}\left(\left\{x: \widehat{\pi}_{(x)_{m}}^{(m)} \Longrightarrow \delta_{\theta}\right\} \cap g L\right)=P_{\theta_{0}}\left(g^{-1}\left(\left\{x: \widehat{\pi}_{(x)_{m}}^{(m)} \Longrightarrow \delta_{\theta}\right\} \cap g L\right)\right) \\
& \quad=P_{\theta_{0}}\left(\left\{x: \widehat{\pi}_{(g x)_{m}}^{(m)} \Longrightarrow \delta_{\theta}\right\} \cap L\right)=P_{\theta_{0}}\left(\left\{x: \widehat{\pi}_{(x)_{m}}^{(m)}\left(\bar{g}^{-1} \cdot\right) \Longrightarrow \delta_{\theta}\right\} \cap L\right) \\
& \quad=P_{\theta_{0}}\left(\left\{x: \widehat{\pi}_{(x)_{m}}^{(m)} \Longrightarrow \delta_{\theta_{0}}\right\} \cap L\right)=1 .
\end{aligned}
$$

Наконец, пусть $r$ - ограниченная непрерывная положительная функция на $\Theta$ и $\rho(B)=\int_{B} r(\theta) \pi(d \theta), B \in \mathscr{B}$. Тогда для любого целого $m \geqslant m_{0}$ и любого $x=\left(x_{1}, x_{2}, \ldots\right)$ из $\mathscr{X}^{\infty}$ формула

$$
\widehat{\rho}_{(x)_{m}}^{(m)}(B)=\frac{\int_{B} r(\theta) \widehat{\pi}_{(x)_{m}}^{(m)}(d \theta)}{\int r(\theta) \widehat{\pi}_{(x)_{m}}^{(m)}(d \theta)}
$$

определяет версию апостериорного распределения при заданных $X_{1}=$ $x_{1}, \ldots, X_{m}=x_{m}$ относительно априорной меры $\rho$. Из этой формулы и из самого определения слабой сходимости вытекает, что состоятельность при некотором $\theta$ из $\Theta$ для версий апостериорных распределений $\widehat{\pi}_{(\cdot)_{m}}^{(m)}(\cdot)$ относительно априорной меры $\pi$ влечет состоятельность при этом $\theta$ для версий апостериорных распределений $\rho_{(\cdot)_{m}}^{(m)}(\cdot)$ относительно априорной меры $\rho$.

3 а м е ч а н и е 3.3 . В условии (ii) теоремы $3.1 \widehat{\pi}^{(m)}(\cdot)$ можно определить эквивалентным образом как $(G-\bar{G})$-инвариантное марковское ядро на $\left(\mathscr{X}^{m}, \mathscr{A}_{c}^{m}\right) \times \mathscr{B}$, такое, что $\widehat{\pi}^{(m)}(B)=\pi^{(m)}(B) Q_{m}$-п.в. для любого множества $B$ из $\mathscr{B}$. 
3 а м е ч а н и е 3.4. Оказывается, что при выполнении дополнительных ограничений (выполняющихся в ряде важных для приложений случаев) можно указать условие в терминах $P_{\theta}$ для одного лишь фиксированного $\theta=\theta_{0}$, которого достаточно для выполнения условия (i), когда семейство $\left\{P_{\theta}: \theta \in \Theta\right\} G$-инвариантно. Сохраняя обозначения, введенные в замечании 2.2 (см. первые два и предпоследний абзацы этого замечания), предположим, что $\mathscr{X}$ есть польское пространство, $D=\mathscr{P}$, $T(\theta)=P_{\theta}^{(1)}$, семейство $\left\{P_{\theta}: \theta \in \Theta\right\} G$-инвариантно, отображения $x \rightarrow g x$ непрерывны для всех $g$ из $G$ и группа $\bar{G}$ действует транзитивно на $\Theta$. Пусть $T_{n}, n \geqslant 1,-$ измеримое отображение пространства $\left(\mathscr{X}^{n}, \mathscr{A}^{n}\right)$ в $D=\mathscr{P}$ такое, что $T_{n}(g x)(\cdot)=T_{n}(x)\left(g^{-1} \cdot\right)$ при всех $g$ из $G$ и $x$ из $\mathscr{X}^{n} . \mathrm{B}$ частности, можно взять $T_{n}(x)=(1 / n) \sum_{1}^{n} \delta_{x_{i}}$ для $x=\left(x_{1}, \ldots, x_{n}\right)$. Тогда условие

$\lim _{n} P_{\theta_{0}}\left(x: d\left(T_{n}\left(x_{(n)}\right), P_{\theta_{0}}^{(1)}\right)>\varepsilon\right)=1$ для некоторого $\theta_{0} \in \Theta$ и всех $\varepsilon>0$

влечет (2.5). Действительно, возьмем подпоследовательность $\left\{n^{\prime}\right\}$ последовательности натуральных чисел такую, что

$$
P_{\theta_{0}}\left(x: T_{n^{\prime}}\left(x_{\left(n^{\prime}\right)}\right) \Longrightarrow P_{\theta_{0}}^{(1)}\right)=1
$$

и для произвольного $\theta$ из $\Theta$ пусть $\bar{g}$ из группы $\bar{G}$ таково, что $\theta=\bar{g} \theta_{0}$. Тогда, рассуждая как в (3.6) (и заменяя, для простоты, $n^{\prime}$ на $n$ ), имеем

$$
\begin{aligned}
P_{\theta}\left(x: T_{n}\left(x_{(n)}\right) \longrightarrow P_{\theta}^{(1)}\right) & =P_{\theta_{0}}\left(x: g^{-1}\left(T_{n}\left(x_{(n)}\right) \Longrightarrow P_{\theta}^{(1)}\right)\right) \\
& \left.=P_{\theta_{0}}\left(x: T_{n}\left(g x_{(n)}\right) \Longrightarrow P_{\theta}^{(1)}\right)\right) \\
& =P_{\theta_{0}}\left(x: T_{n}\left(x_{(n)}\right)\left(g^{-1} \cdot\right) \Longrightarrow P_{\theta_{0}}^{(1)}\left(g^{-1} \cdot\right)\right) \\
& \left.=P_{\theta_{0}}\left(x: T_{n}\left(x_{(n)}\right) \Longrightarrow P_{\theta_{0}}^{(1)}\right)\right)=1,
\end{aligned}
$$

как и утверждалось. Таким образом, согласно утверждению $\left({ }^{*}\right)$ из замечания 2.2 , условие (i) выполнено, если также $\mathscr{B} \subset \mathscr{B}_{1}$.

3 а м е ч а н и е 3.5 . Теорема 3.1 остается справедливой, если в условии (ii) марковские ядра $\widehat{\pi}^{(m)}(\cdot)(G-\bar{G})$-инвариантны лишь на некоторых $G$-инвариантных подмножествах $E_{m}$ пространств $\mathscr{X}^{m}$ таких, что $Q_{m}\left(E_{m}^{c}\right)=0$.

4. Существование инвариантных марковских ядер. 4.а. Общий случай. Напомним, что линейным лифтингом на пространстве $M_{Z}^{\infty}=M^{\infty}(Z, \mathscr{Z}, \mu)$ действительных ограниченных измеримых функций, определенных на измеримом пространстве $(Z, \mathscr{Z}, \mu)$, называется отображение $l$ пространства $M_{Z}^{\infty}$ в себя такое, что для любых $f, f_{1}, f_{2}$ из $M_{Z}^{\infty}$ и любых действительных $c_{1}, c_{2}$

(1) $l(f)=f \mu$-п.в.,

(2) $f_{1}=f_{2} \mu$-п.в. влечет $l\left(f_{1}\right)=l\left(f_{2}\right)$,

(3) $f \geqslant 0$ влечет $l(f) \geqslant 0$, 
(4) $l(1)=1$,

(5) $l\left(c_{1} f_{1}+c_{2} f_{2}\right)=c_{1} l\left(f_{1}\right)+c_{2} l\left(f_{2}\right)$.

Предположим, что мера $Q_{m}$ доминирована $\bar{R}^{+}$-значной мерой $q$ на $\left(\mathscr{X}^{m}, \mathscr{A}^{m}\right)$ и обозначим $\mathscr{A}_{c}^{m}$ область определения пополнения $q_{c}$ меры $q$. Если $H$ есть группа, действующая слева на некотором множестве $Z$, то для любой действительной функции $f$ на $Z$ и любого элемента $h$ из $H$ определим $h f$, положив $h f(z)=f(h z), z \in Z$. Пусть пространство $\Theta$ локально компактно, $m$ таково, что мера $Q_{m} \sigma$-конечна и $\pi .^{(m)}(\cdot)$ - некоторое фиксированное марковское ядро из леммы 2.1.

Предложение 4.1. Если существует линейный лифтинг $l$ на $M_{\mathscr{X}^{m}}^{\infty}=M^{\infty}\left(\mathscr{X}^{m}, \mathscr{A}_{c}^{m}, q_{c}\right)$, коммутирующий $c G($ m.e. $l(g f)=g l(f) \partial \Omega я$ всех $g$ из $G$ uf из $\left.M_{\mathscr{X}^{m}}^{\infty}\right)$, то существует $(G-\bar{G})$-инвариантная версия марковского ядра $\pi^{(m)}(\cdot)$ в следующем смысле: существует $(G-\bar{G})$ инвариантное марковское ядро $k^{(m)}(\cdot)$ на $\left(\mathscr{X}^{m}, \mathscr{A}_{c}^{m}\right) \times \mathscr{B}$ такое, ито для некоторого множества $N$ из $\mathscr{A}^{m}$ имеем $Q_{m}(N)=0 u$

$$
k_{x}^{(m)}(\cdot)=\pi_{x}^{(m)}(\cdot), \quad \text { если } x \notin N .
$$

Д о к а з а т е л ь с т в о. Доказательство основано на идее использованной Ле Камом в [17] (см. также предложение 8.1 в [18]). Для упрощения обозначений мы будем опускать индекс $m$ в $Q_{m}, \pi r^{(m)}(\cdot)$ и $k^{(m)}(\cdot)$ на протяжении всего доказательства. Пусть, как и выше, $C_{0}=C_{0}(\Theta)$ обозначает класс всех действительных непрерывных функций на $\Theta$ c компактным носителем. Тогда для любого фиксированного элемента $x$ пространства $\mathscr{X}^{m}$ отображение

$$
f \in C_{0} \longrightarrow\left(l \circ \int f(\theta) \pi \cdot(d \theta)\right)(x)
$$

есть положительный линейный функционал на $C_{0}$. Следовательно, по теореме Рисса-Маркова, существует единственная борелевская мера на $\mathscr{B}$, обозначим ее $k_{x}(\cdot)$, такая, что

$$
\left(l \circ \int f(\theta) \pi \cdot(d \theta)\right)(x)=\int f(\theta) k_{x}(d \theta) \stackrel{\text { def }}{=} k_{x}(f) .
$$

Далее, в силу коммутативности $l$ и $G$ и $\left(3.2^{*}\right)$, для всех $g$ из $G$ и $f$ из $C_{0}$ имеем:

$$
\begin{aligned}
& k_{g x}\left(\bar{g}^{-1} f\right)=\int f\left(\bar{g}^{-1} \theta\right) k_{g x}(d \theta)=\left(l \circ \int f\left(\bar{g}^{-1} \theta\right) \pi \cdot(d \theta)\right)(g x) \\
& =\left(l \circ \int f\left(\bar{g}^{-1} \theta\right) \pi_{g} \cdot(d \theta)\right)(x)=\left(l \circ \int f(\theta) \pi \cdot(d \theta)\right)(x)=k_{x}(f) .
\end{aligned}
$$

Применяя формулу замены переменной в интеграле и используя единственность в теореме Рисса-Маркова, мы выводим, что $k_{g x}(\bar{g} B)=$ $k_{x}(B)$ для всех $g$ из $G, x$ из $\mathscr{X}^{m}$ и $B$ из $\mathscr{B}$. Кроме того, из (4.1) вытекает, что $\pi .(f)=k .(f) q$-п.в. для любой функции $f$ из $C_{0}$. Поскольку $C_{0}$, как 
отмечалось выше, сепарабельно в равномерной норме и поскольку существует последовательность $\left\{f_{n}\right\}$ неотрицательных функций из $C_{0}$, для которой $f_{n} \uparrow 1$ (это влечет, что $k_{x}(\cdot)$ есть вероятностная мера $q$-п.в.), то существует множество $N$ в $\sigma$-алгебре $\mathscr{A}^{m}$ для которого $q(N)=0$ и такое, что $k_{x}(f)=\pi_{x}(f)$, если $x \notin N$ при всех $f$ из $C_{0}$. Теперь для завершения доказательства остается лишь еще раз применить единственность в теореме Рисса-Маркова и использовать доминированность меры $Q$ мерой $q$.

Предложение 4.1 приводит к вопросу: когда существует лифтинг коммутирующий с группой преобразований? Вот известные нам общие результаты, дающие для этого достаточные условия.

(I) В [13] доказано, что в локально компактной группе с полной левой мерой Хаара существует лифтинг, коммутирующий с левыми сдвигами.

(II) Из теоремы 3.7 в [14] вытекает, что если $\mathscr{Z}$ - компактное хаусдорфово пространство и $H-$ группа действующая слева на $\mathscr{Z}$, такие, чTO

(a) если $z_{1} \neq z_{2}, z_{1}, z_{2} \in \mathscr{Z}$, то не существует сети $\left\{h_{\alpha}\right\}$ в $H$ такой, что $\lim _{\alpha} h_{\alpha} z_{1}=\lim _{\alpha} h_{\alpha} z_{2}$,

(b) орбита $\{h z: h \in H\}$ любого элемента $z$ из $\mathscr{Z}$ плотна в $\mathscr{Z}$, то существует $H$-инвариантная полная радоновская мера $\mu$ на $\mathscr{Z}$ такая, что на пространстве $M^{\infty}(\mathscr{Z}, \mu)$ есть линейный лифтинг, коммутирующий с $H$. Ясно, что если существует лишь одна $H$-инвариантная полная радоновская мера $\mu$ на $\mathscr{Z}$, то на пространстве $M^{\infty}(\mathscr{Z}, \mu)$ есть линейный лифтинг, коммутирующий с $H$. Отметим также, что из теоремы 25 главы 14 в [24] следует, что на $\mathscr{Z}$ существует единственная $H$-инвариантная полная радоновская мера, если $H-$ топологическая группа, действующая на $\mathscr{Z}$ транзитивно и правильно (правильно в том смысле, что прообразы относительно отображений $h \rightarrow h z$ компактных множеств из $\mathscr{Z}$ компактны в $H)$.

\section{4.b. Случай доминированного семейства и независимых на-}

блюдений. Сохраняя введенные выше обозначения, предположим, что наблюдения $X_{1}, X_{2}, \ldots$ независимы и одинаково распределены и что вероятностные меры $P_{\theta}^{(1)}$ доминированы $\sigma$-конечной мерой $\nu$ на $\mathscr{A}$, обладающей свойством относительной $G$-инвариантности с мультипликатоpoм $\chi$, т.е.

$$
\int f\left(g^{-1} x\right) \nu(d x)=\chi(g) \int f(x) \nu(d x) \text { для всех } g \in G,
$$

для всех неотрицательных $\mathscr{A}$-измеримых функций $f$. Под мультипликатором мы понимаем гомоморфизм $\chi$ группы $G$ в $\mathbf{R}^{+}=(0, \infty)$ (т.е. функцию $\chi$, определенную на $G$ и принимающую значения из $\mathbf{R}^{+}$, такую, что $\chi(g h)=\chi(g) \chi(h)$ при всех $g$ и $h$ из $G)$.

3 а м е ч а н и е 4.1. Известно, что в некоторых частных случаях абсолютная непрерывность мер $P_{\theta}^{(1)}, \theta \in \Theta$, относительно некоторой 
$\sigma$-конечной меры влечет их непрерывность относительно инвариантной меры (см. [9] для случая семейства сдвигов распределения на прямой и [21] для случая инвариантного относительно вращений семейства распределений на единичной сфере в $\mathbf{R}^{k}$ ). При некоторых дополнительных ограничениях подобный феномен имеет место и для общей $G$-инвариантной вероятностной модели.

Действительно, пусть $\left\{P_{\theta}^{\prime}: \theta \in \Theta\right\}-G$-инвариантная вероятностная модель на $(\mathscr{X}, \mathscr{A})$ (т.е. $G$ есть группа, действующая слева на $\mathscr{X}$, и для любых $g$ из $G$ и $\theta$ из $\Theta$ существует единственный элемент из $\Theta$, обозначаемый $\bar{g} \theta$, такой, что $P_{\theta}^{\prime}\left(g^{-1} A\right)=P_{\bar{g} \theta}^{\prime}(A)$ для всех $A$ из $\left.\mathscr{A}\right)$ такая, что мера $P_{\theta}^{\prime}$ для каждого $\theta$ доминирована $\sigma$-конечной мерой $\alpha$ на $\mathscr{A}$. Более того, предположим, что

$(\alpha)$ существует единственная $\sigma$-конечная мера $\nu^{\prime}$ на $\mathscr{A}$, обладающая свойством относительной $G$-вариантности, с мультипликатором $\chi$,

$(\beta)$ на некоторой $\sigma$-алгебре $\mathscr{G}$ подмножеств $G$ такой, что отображение $g \rightarrow g h$ измеримо для любого $h$ из $G$, существует относительно право $G$-инвариантная $\sigma$-конечная мера $\lambda$ с мультипликатором $\chi^{-1}$ (т.е. $\int f\left(g h^{-1}\right) \lambda(d g)=\chi^{-1}(h) \int f(g) \lambda(d g)$ для всех неотрицательных $\mathscr{G}$-измеримых функций $f$ и всех $h$ из $G$ ),

$(\gamma)$ отображение $(x, g) \rightarrow g^{-1} x$ измеримого пространства $(\mathscr{X} \times$ $G, \mathscr{A} \times \mathscr{G})$ в измеримое пространство $(\mathscr{X}, \mathscr{A})$ измеримо.

(Эти условия выполнены в указанных выше частных случаях, а также в моделях, рассматриваемых в примерах 5.1-5.3 ниже.)

Тогда вероятности $P_{\theta}^{\prime}$ абсолютно непрерывны относительно $\nu^{\prime}$ при всех $\theta$ из $\Theta$.

Чтобы в этом убедиться допустим, не ограничивая общности, что $\alpha(\mathscr{X})<\infty$ и что, напротив, $P_{\theta}^{\prime}(E)>0$ и $\nu^{\prime}(E)=0$ для некоторых $\theta$ и $E$. Тогда $P_{\bar{g} \theta}^{\prime}(g E)=P_{\theta}^{\prime}(E)>0$ для любого $g$ из $G$, и, следовательно, $\alpha(g E)>0$ для всех $g$ из $G$. Далее, в силу условия $(\gamma)$ и теоремы Фубини,

$$
\int_{G} \alpha(g E) \lambda(d g)=\int_{G} \int_{\mathscr{X}} 1_{E}\left(g^{-1} x\right) \alpha(d x) \lambda(d g)=\int_{\mathscr{X}} \nu_{x}(E) \alpha(d x),
$$

где для любых $x$ из $\mathscr{X}, A$ из $\mathscr{A}$ и $h$ из $G$, согласно условию $(\beta), \nu_{x}(h A)=$ $\int_{G} 1_{A}\left((g h)^{-1} x\right) \lambda(d g)=\chi(h) \nu_{x}(A)$. По условию $(\alpha)$ должно быть $\nu_{x}=\nu^{\prime}$ при всех $x$ из $\mathscr{X}$ и, следовательно,

$$
0<\int_{G} \alpha(g E) \lambda(d g)=\nu^{\prime}(E) \alpha(\mathscr{X})=0
$$

Полученное противоречие доказывает наше утверждение.

Пусть $p(\cdot, \theta)$ есть плотность $P_{\theta}^{(1)}$ относительно $\nu$. Тогда функция $p(x, \theta)$ почти $(G-\bar{G})$-инвариантна, т.е. для любых $g$ из $G$ и $\theta$ из $\Theta$

$$
p(x, \theta)=p(g x, \bar{g} \theta) \chi(g) \quad \nu \text {-п.в. }
$$

(см. [8], раздел 7.3), причем множество $\nu$-меры нуль, где (4.3) не выполняется, в общем случае может зависеть и от $g$ и от $\theta$. Однако, 
как отмечается в $i b i d$. , в большинстве приложений существуют версии плотностей $p(x, \theta)$, для которых (4.3) выполняется всюду, и семейство $\{p(x, \theta), \theta \in \Theta\}$ таких версий называется $(G-\bar{G})$-инвариантным.

Мы приведем теперь некоторые общие достаточные условия для существования $(G-\bar{G})$-инвариантной функции $q(x, \theta)$, совпадающей с $p(x, \theta)(\nu \times \pi)$-п.в. (это именно то, что нужно ниже для наших целей). Предположим, что функция $p(x, \theta)(\mathscr{A} \times \mathscr{B})$-измерима. Мы можем рассматривать $G$ как группу преобразований, действующую слева на $\mathscr{X} \times \Theta$, положив $g(x, \theta)=(g x, \bar{g} \theta)$. Измеримость $p(x, \theta), g$ и $\bar{g}$ (как отображений $x \rightarrow g x$ и $\theta \rightarrow \bar{g} \theta$ соответственно) влечет $(\mathscr{A} \times \mathscr{B})$-измеримость $p(g x, \bar{g} \theta)$ для любого фиксированного $g$ из $G$. Поэтому для любого $g$ из $G$

$$
\{(x, \theta): p(x, \theta)=p(g x, \bar{g} \theta) \chi(g)\} \in \mathscr{A} \times \mathscr{B},
$$

и, следовательно, в силу (4.3) и теоремы Фубини, для любого $g$ из $G$

$$
p(x, \theta)=p(g x, \bar{g} \theta) \chi(g) \quad(\nu \times \tau) \text {-п.в., }
$$

где $\tau$ - любая $\sigma$-конечная мера на $\Theta$, т.е. функция $p$ почти $G$-инвариантна с мультипликатором $\chi$ на $\mathscr{X} \times \Theta$ относительно $\nu \times \tau$. Теперь мы используем следующее обобщение теоремы 4 (именуемой некоторыми исследователями теоремой Стейна) из раздела 6.5 в [19].

Предложение 4.2. Пусть $(\mathscr{Y}, \mathscr{C}, \mu)-$ пространство $c$ б-конечной мерой $\mu$ u $(G, \mathscr{G})$ - измеримое пространство, где $G-$ группа, действующая слева на Я્У. Предположим, что отображение $(y, g) \rightarrow g y$ пространства $(\mathscr{Y} \times G, \mathscr{C} \times \mathscr{G})$ в пространство $(\mathscr{Y}, \mathscr{C})$ измеримо и что существует $\sigma$-конечная мера $\lambda$ на $\mathscr{G}$ такая, что $\lambda(E)=0$ влечет $\lambda(E g)=0$ при всех $g$ из $G$ (т.е. $\lambda$ квази-инвариантна справа). Пусть $\varphi-$ измеримая почти $G$-инвариантная с мультипликатором $\chi$ функиия на $\mathscr{Y}$, т.е. такая, что для любого $g$ из $G$

$$
\varphi(y)=\varphi(g y) \chi(g) \quad \mu-n . в .
$$

Тогда если интеграл $\int \varphi(g y) \lambda(d g)$ имеет смысл для всех у из $\mathscr{Y}$ и мультипликатор ұ измерим, то существует $\mathscr{C}$-измеримая $G$-инвариантная с мультипликатором $\chi$ функиия $\psi$ эквивалентная $\varphi$, т.е. для всех $g$ из $G$ и у из $\mathscr{Y}$

$$
\psi(y)=\psi(g y) \chi(g) \quad u \quad \psi(y)=\varphi(y) \quad \mu-n .8 .
$$

Д о к а з а т е ль с т в о. Доказательство проводится по той же схеме, что и доказательство упомянутой выше теоремы из [19].

Из теоремы Фубини вытекает, что $\varphi(y)=\varphi(g y) \chi(g) \lambda$-п.в. для всех $y$, не принадлежащих некоторому множеству $N \mu$-меры нуль. Без ограничения общности мы можем предположить, что $\int \chi\left(g^{-1}\right) \lambda(d g)=1$. Определим множество $A$ (принадлежащее $\sigma$-алгебре $C$ ), положив

$$
A=\left\{y: \int_{G} \varphi\left(g^{\prime} y\right) \lambda\left(d g^{\prime}\right)=\varphi(g y) \chi(g) \quad \lambda \text {-п.в. }\right\}
$$


и пусть

$$
\psi(y)= \begin{cases}\int_{G} \varphi(g y) \lambda(d g), & \text { если } y \in A \\ 0, & \text { если } y \notin A .\end{cases}
$$

Тогда $y \notin N$ влечет $y \in A$ и $\psi(y)=\varphi(y)$.

Далее, для произвольного элемента $y$ из $\mathscr{Y}$ положим

$$
N_{y}^{c}=\left\{g: \int_{G} \varphi\left(g^{\prime} y\right) \lambda\left(d g^{\prime}\right)=\varphi(g y) \chi(g)\right\}
$$

и для любого элемента $h$ из $G$ обозначим $N_{h, y}^{c}=N_{y}^{c} \cap N_{y}^{c} h^{-1}$. Тогда для любых элементов $g$ из $N_{h, y}^{c}$ и $h$ из $G$

$$
\varphi(g h y) \chi(g h)=\int_{G} \varphi\left(g^{\prime} y\right) \lambda\left(d g^{\prime}\right)=\varphi(g y) \chi(g) \text {. }
$$

Выберем и зафиксируем какой-либо элемент $y$ из $A$. Тогда, очевидно, $\lambda\left(N_{y}\right)=0$ и, в силу правой квазиинвариантности $\lambda, \lambda\left(N_{h, y}\right)=0$, так что для любого элемента $g$ из $N_{h, y}^{c}$

$$
\begin{aligned}
\int_{G} \varphi\left(g^{\prime} h y\right) \lambda\left(d g^{\prime}\right) & =\int_{N_{h, y}^{c}} \varphi\left(g^{\prime} y\right) \chi^{-1}(h) \lambda\left(d g^{\prime}\right) \\
& =\varphi(g y) \chi(g) \chi^{-1}(h)=\varphi(g h y) \chi(g) .
\end{aligned}
$$

Следовательно, $h y \in A$, и из первой строки предыдущей формулы вытекает, что $\psi(y)=\psi(h y) \chi(h)$ для всех элементов $h$ из $G$ и $y$ из $A$.

Полагая теперь в предложении $4.2(\mathscr{Y}, \mathscr{C}, \mu)=(\mathscr{X} \times \Theta, \mathscr{A} \times \mathscr{B}, \nu \times \tau)$, $\varphi(y)=p(x, \theta)$, получаем следующее предложение.

Предложение 4.3. Пусть плотность $p(x, \theta)(\mathscr{A} \times \mathscr{B})$-измерима $u$ пусть соотношение (4.3) выполняется с измеримьм мультипликатором $\chi$. Тогда если существует $\sigma$-конечная мера $\lambda$, определенная на некоторой $\sigma$-алгебре $\mathscr{G}$ подмножеств $G$ такая, что $\lambda(E)=0$ влечет $\lambda(E g)=0$ при всех $g$ из $G, u$, если отображение $(x, \theta, g) \rightarrow(g x, \bar{g} \theta)$ пространства $(\mathscr{X} \times \Theta \times G, \mathscr{A} \times \mathscr{B} \times \mathscr{G})$ в пространство $(\mathscr{X} \times \Theta, \mathscr{A} \times \mathscr{B})$ измеримо, то существует $(\mathscr{A} \times \mathscr{B})$-измеримая $(G-\bar{G})$-инвариантная с мультипликатором х функиия $q(x, \theta)$ (т.е. такая, что $q(x, \theta)=$ $q(g x, \bar{g} \theta) \chi(g)$ при всех $x$ из $\mathscr{X}, \theta$ из $\Theta$ и $g$ из $G)$, для которой

$$
q(x, \theta)=p(x, \theta) \quad(\nu \times \tau)-\text { n.в. }
$$

Предположим теперь, что существует функция $q(x, \theta)$, обладающая свойствами указанными в предложении $4.3 \mathrm{c} \tau=\pi$.

Предложение 4.4. Множества

$$
A_{m}=\left\{x=\left(x_{1}, \ldots, x_{m}\right) \in \mathscr{X}^{m}: I_{m}(x) \stackrel{\text { def }}{=} \int\left(\prod_{1}^{m} q\left(x_{i}, \theta\right)\right) \pi(d \theta)<\infty\right\} .
$$

$m=1,2, \ldots G$-инвариантны и формула

$$
\pi_{x}^{(m)}(B)=\frac{\int_{B}\left(\prod_{1}^{m} q\left(x_{i}, \theta\right)\right) \pi(d \theta)}{\int\left(\prod_{1}^{m} q\left(x_{i}, \theta\right)\right) \pi(d \theta)}
$$


определяет $(G-\bar{G})$-инвариантное марковское ядро на $\left(A_{m}, \mathscr{A}^{m} \cap A_{m}\right) \times$ $\mathscr{B}$.

Кроме того, если для некоторого натурального $m_{1}$

$$
\nu^{m_{1}}\left(A_{m_{1}}^{c}\right)=0
$$

mo $\nu^{m}\left(A_{m}^{c}\right)=0$ nри всех $m \geqslant m_{1}$.

Д о к а з а т е л ь с т в о. Обозначим $I_{m}(x, B)$ числитель в правой части формулы (4.5). Тогда в силу $(G-\bar{G})$-инвариантности $q(\cdot, \cdot)$ и $G$-инвариантности $\pi$ для любого натурального $m$ и любого $g$ из $G$

$$
\begin{aligned}
I_{m}(g x) & =(\chi(g))^{-m} I_{m}(x), \\
I_{m}(g x, \bar{g} B) & =(\chi(g))^{-m} I_{m}(x, B) .
\end{aligned}
$$

Следовательно, множества $A_{m} G$-инвариантны, а функция $\pi^{(m)}(\cdot)$ $(G-\bar{G})$-инвариантна на $A_{m} \times \mathscr{B}$ и, очевидно, является марковским ядром на $\left(A_{m}, \mathscr{A}^{m} \cap A_{m}\right) \times \mathscr{B}$.

Наконец, обозначим для краткости $x^{\prime}=\left(x_{1}, \ldots, x_{m_{1}}\right), x^{\prime \prime}=$ $\left(x_{m_{1}+1}, \ldots, x_{m}\right)$, так что $x=\left(x^{\prime}, x^{\prime \prime}\right)$, и отметим, что если $x^{\prime}$ принадлежит $A_{m_{1}}$, то при $m \geqslant m_{1}$

$$
\int I_{m}(x) \nu^{m-m_{1}}\left(d x^{\prime \prime}\right)=I_{m_{1}}\left(x^{\prime}\right)<\infty .
$$

Отсюда вытекает, что для таких $x^{\prime}$

$$
\nu^{m-m_{1}}\left(\left(A_{m}^{c}\right)_{x^{\prime}}\right)=0,
$$

где $\left(A_{m}^{c}\right)_{x^{\prime}}$ обозначает $x^{\prime}$-сечение $A_{m}^{c}$. Применяя теперь теорему Фубини из (4.6) и (4.7), выводим

$$
\nu^{m}\left(A_{m}^{c}\right)=\int_{A_{m_{1}}^{c}} \nu^{m-m_{1}}\left(\left(A_{m}^{c}\right)_{x^{\prime}}\right) \nu^{m_{1}}\left(d x^{\prime}\right)=0,
$$

как и утверждалось.

Следствие 4.1. Пусть при $m=1,2, \ldots$

$$
\widehat{\pi}_{x}^{(m)}(B)= \begin{cases}\pi_{x}^{(m)}(B), & \text { если } x \in A_{m}, \\ \widehat{p}(B), & \text { если } x \notin A_{m},\end{cases}
$$

где $\pi^{(m)}(\cdot)$ определено в (4.5) и $\widehat{p}$ - некоторая вероятностная мера на $(\Theta, \mathscr{B})$. Тогда если

$$
Q_{m}\left(A_{m}^{c}\right)=0
$$

где $Q_{m}$ может быть записано в виде

$$
Q_{m}(A)=\int_{\Theta} \int_{A}\left(\prod_{1}^{m} p\left(x_{i}, \theta\right)\right) \nu^{m}(d x) \pi(d \theta),
$$

то $\widehat{\pi}^{(m)}(\cdot)$ есть $(G-\bar{G})$-инвариантная на $G$-инвариантном множестве $A_{m}$ дезынтеграчия меры $\mu_{m}$ относительно $Q_{m}$. Более того, мера $Q_{m} \sigma$-конечна при всех $m$ и при выполнении (4.6) $Q_{m}\left(A_{m}^{c}\right)=0$ при всех $m \geqslant m_{1}$. 
Д о к а з а т е л с т в о. Стандартное вычисление показывает, что при выполнении $(4.8) \widehat{\pi}^{(m)}(\cdot)$ действительно есть дезынтеграция $\mu_{m}$ относительно $Q_{m}$. Остальные утверждения немедленно вытекают из выписанной выше формулы для $Q_{m}$ и предложения 4.4.

5. Частные случаи. П р и м е р 5.1. Пусть $\mathscr{X}=G=\Theta=\mathscr{I}$, где $\mathscr{I}$ - локально-компактная группа, и предположим также, в соответствии с нашими общими допущениями, что $\mathscr{I}$ есть борелевское подмножество польского пространства. Действие $g x$ элемента $g$ из $G$ на элемент $x$ из $\mathscr{X}$ определяется как произведение в $\mathscr{I}$ и $\mathscr{A}$ обозначает борелевскую $\sigma$-алгебру в $\mathscr{X}$. Пусть $P$ - вероятностная мера на $\left(\mathscr{X}^{\infty}, \mathscr{A}^{\infty}\right)$, определим $P_{\theta}(A)=P\left(\theta^{-1} A\right)$ для множеств $A$ из $\mathscr{A}^{\infty}$ и предположим, что меры $P_{\theta}$ различны для различных $\theta$ из $\Theta$ (когда, например, $\mathscr{X}=\mathbf{R}^{k}$ любая вероятностная мера на $\mathscr{A}^{\infty}$ удовлетворяет этому условию, однако когда $\mathscr{X}^{\infty}$ имеет компактные подгруппы, мера $P$, сосредоточенная на компактной подгруппе и левоинвариантная на ней не удовлетворяет этому условию). Очевидно,

$$
P_{\theta}\left(g^{-1} A\right)=P_{g \theta}(A), \quad \text { при всех } \theta \in \Theta, \quad g \in G, A \in \mathscr{A}^{\infty},
$$

так что в рассматриваемом случае преобразование $g$ есть просто левый сдвиг на $g$ в $\Theta=\mathscr{I}$. В качестве $\pi$ мы берем здесь левоинвариантную меру Хаара. Обозначая теперь $\left.P^{(m)}\right)$ образ $P$ при проектировании $x=$ $\left(x_{1}, \ldots\right) \rightarrow\left(x_{1}, \ldots, x_{m}\right)$, имеем для любого натурального $m$ и любого множества $A$ из $\mathscr{A}^{m}$

$$
\begin{aligned}
Q_{m}(A) & =\int P^{(m)}\left(\theta^{-1} A\right) \pi(d \theta)=\iint 1_{A}(\theta x) P^{(m)}(d x) \pi(d \theta) \\
& =\iint 1_{A x^{-1}}\left(\theta e_{m}\right) \pi(d \theta) P^{(m)}(d x),
\end{aligned}
$$

где $e_{m}$ есть единица в $G^{m}$. Из этого выражения легко следует, что мера $Q_{m}$ конечна на компактных множествах и, следовательно, $\sigma$-конечна, поскольку группа $G \sigma$-компактна. Предположим также, что

$$
\text { мера } Q_{m} \text { доминирована мерой } \pi^{m}, \quad m=1,2, \ldots,
$$

и отметим, что $\pi^{m}$ есть мера Хаара на $G^{m}$. Тогда по теореме Ионеску Тулчи [13] условие предложения 4.1 выполняется с $q=\pi^{m}$ и, следовательно, выполняется также условие (ii) теоремы 3.1. Отсюда вытекает, поскольку условие (iii) этой теоремы верно в рассматриваемом случае, то для справедливости утверждения теоремы 3.1 достаточно выполнения ее условия (i). Отметим, что согласно замечанию 2.2 условие (i) выполняется, например, если $\mathscr{X}$ метризуемо как польское пространство и наблюдения $X_{1}, X_{2}, \ldots$ подчиняются усиленному закону больших чисел (2.7) для $\pi$-почти всех $\theta$ из $\Theta$ (или если условие (3.7) справедливо) при выполнении включения $\mathscr{B} \subset \mathscr{B}_{1}$, и это включение имеет место в рассматриваемом случае, если отображение $\theta \rightarrow T(\theta)$ инъективно. 
Резюмируя вышесказанное, мы видим, что в случае, рассматриваемом в настоящем примере, утверждение теоремы 3.1 справедливо, если выполняется (5.1) вместе с условием (i), сформулированном в теореме 2.1. Это условие (i) выполнено, например, при инъективности отображения $\theta \rightarrow T(\theta)$, если $\mathscr{X}$ метризуемо как польское пространство и $X_{1}, X_{2}, \ldots$ подчиняются усиленному закону больших чисел $(2.7)$ для $\pi$-почти всех $\theta$ из $\Theta$ (в частности, когда $X_{1}, X_{2}, \ldots$ н.о.р. или строго стационарны и эргодичны и $\left.T(\theta)=P_{\theta}^{(1)}\right)$ или, если выполнено условие (3.7).

Для выполнения условия (ii) в рамках настоящего примера мы наложили дополнительно ограничение (5.1), которое, очевидно, выполняется, если мера $P^{(m)}$ доминирована мерой $\pi^{m}, m=1,2, \ldots$. Чтобы избавиться от этого ограничения при применении используемого нами метода потребовалась бы справедливость предложения 4.1 с $\mathscr{X}=G=\mathscr{I}$ и $q=Q_{m}$, однако мы не располагаем доказательством этого предложения при таких $\mathscr{X}$ и $q$.

З а м е ч а н и е 5.1. В работе Ло [20] изучалась байесовская состоятельность для модели сдвига на прямой, что соответствует $\mathscr{X}=G=$ $\Theta=\mathbf{R}^{1}$ в нашем примере 5.1. Им дано достаточное условие байесовской состоятельности как некоторое условие измеримости, однако оно отличается от нашего условия (i), будучи выраженным в терминах специальной фильтрации вспомогательных $\sigma$-алгебр, тесно связанной с формой выбранных им марковских ядер $\pi_{.^{(m)}}(\cdot)$. Используемая Ло, конструкция не обобщается, по-видимому, на обшую инвариантную статистическую модель.

Пусть $\mathscr{X}=S=\left\{x \in \mathbf{R}^{k}:|x|=1\right\}$, где $|\cdot|$ - обычная евклидова норма, и пусть $G$ - группа $\mathscr{O}$ ортогональных преобразований $\mathbf{R}^{k}$.

П р и м е р 5.2а. Рассмотрим семейство $\left\{P_{y}(\cdot): y \in S\right\}$ борелевских вероятностных мер на $S^{\infty}$ такое, что для любых элементов $h$ из $\mathscr{O}, y$ из $S$ и борелевских подмножеств $A$ пространства $S^{\infty}$

$$
P_{h y}(h A)=P_{y}(A),
$$

и предположим, что $P_{y_{1}} \neq P_{y_{2}}$, если $y_{1} \neq y_{2}$. Эти условия выполнены, например, если $P_{y}=\left(P_{y}^{\prime}\right)^{\infty}$, где $P_{y}^{\prime}$ есть вероятностная мера на $S$ с плотностью $f\left(y^{\prime} x\right)$ (здесь $y^{\prime} x$ - скалярное произведение $y$ и $x$ ) относительно равномерного распределения на $S$ и функция $f$ такова, что $\int_{-1}^{1} f(t) t\left(1-t^{2}\right)^{(k-3) / 2} d t \neq 0$, (см., например, формулы (3.4.1), (3.4.2) в [26]). Важным распределением этого типа является $k$-мерное распределение фон Мизеса-Фишера с плотностью $c_{k}(q) \exp \left\{q y^{\prime} x\right\}$, где $q \geqslant 0$,

$$
c_{k}(q)=q^{(k-1) / 2} /(2 \pi)^{k / 2} I_{(k-1) / 2}(q)
$$

и $I_{r}(q)$ обозначает модифицированную функцию Бесселя первого рода порядка $r$. 
В данном случае $\mathscr{X}=\Theta=S, G=\bar{G}=\mathscr{O}$ и, легко видеть, что цитированный в разделе 4 результат Джонсона из [14] применим (в введенных там обозначениях) с $\mathscr{Z}=S^{m}, H=\mathscr{O}^{m}$. Отсюда следует, в силу предложения 4.1, что условие (ii) теоремы 3.1 выполнено. Условие (iii) этой теоремы, очевидно, тоже выполнено и по замечанию 2.2 условие (i) будет также выполнено, например, если $T\left(\theta_{1}\right) \neq T\left(\theta_{2}\right)$ при $\theta_{1} \neq \theta_{2}$ и если наблюдения удовлетворяют условиям указанным в конце примера 5.1 .

3 а м е ч а н и е 5.2. Пример 5.2а показывает, что основное утверждение основной теоремы 3.1 работы Ло и Кабрера [21] (т.е. что (S2) влечет (S3)) вытекает из теоремы 3.1 настоящей статьи. Более того, мы даем обоснование существования $(G-\bar{G})$-инвариантных марковских ядер $\pi^{(m)}(\cdot)$ (распределений Питмана по терминологии Ло-Кабрера) в случае более общем, чем рассматриваемый в [21].

П р и м е р 5.2b. Пусть, как и в предыдущем примере, $\mathscr{X}=S$, $G=\mathscr{O}$ и пусть $P$ - борелевская вероятностная мера на $\mathscr{X}^{\infty}$ такая, что меры $P g^{-1}$ различны для различных $g$ из $G$. Вот простейший случай, когда это так: $\mathscr{X}$ есть единичная окружность $[0,2 \pi)$ и проекция $P^{\prime}$ меры $P$ на одну из компонент пространства $\mathscr{X}^{\infty}$ имеет возрастающую плотность относительно равномерного распределения. Рассмотрим $G$-инвариантную статистическую модель $\left\{P_{g}=P g^{-1}: g \in G\right\}$ (здесь $\left.\Theta=G\right)$. То же рассуждение, что и в примере $5.2 \mathrm{a}$, показывает, что заключение теоремы 3.1 справедливо для этой модели, если выполнено условие (i), сформулированное в теореме 2.1, а для этого, в свою очередь, достаточно, чтобы наблюдения удовлетворяли условиям указанным в конце примера 5.1 и $T\left(\theta_{1}\right) \neq T\left(\theta_{2}\right)$ при $\theta_{1} \neq \theta_{2}$.

П р и м е р 5.3. Пусть $\mathscr{X}=\mathbf{R}^{k}$ и $G=\mathbf{R}^{+} \times \mathbf{R}^{k}$ с групповой операцией в $G$, определенной равенством

$$
\left(a_{1}, x_{1}\right)\left(a_{2}, x_{2}\right)=\left(a_{1} a_{2}, a_{1} x_{2}+x_{1}\right) .
$$

Действие группы $G$ на $\mathscr{X}$ определяется как сжатие и сдвиг: $(a, x) y=$ $a y+x$. Пусть $X-\mathbf{R}^{k}$-значная случайная величина с невырожденным распределением $P^{\prime}$. Для $g=(a, x)$ из $G$ пусть $P_{g}^{\prime}=P^{\prime} g^{-1}$ есть распределение величины $g X=a X+x$. Легко проверить, что $P_{g_{1}}^{\prime} \neq P_{g_{2}}^{\prime}$ при $g_{1} \neq g_{2}$ и что $P_{g}^{\prime} h^{-1}=P_{h g}^{\prime}$ для любого $h$ из $G$. Следовательно, $\left\{P_{g}^{\prime}: g \in G\right)$ есть $G$-инвариантная вероятностная модель и $\left\{P_{g}=\left(P_{g}^{\prime}\right)^{\infty}: g \in G\right\}$ есть $G$-инвариантная статистическая модель с $\Theta=G=\bar{G}$.

Далее, предположим, что $P^{\prime}$ имеет плотность $p$ относительно меры Лебега $\lambda$ на $\mathbf{R}^{k}$. Мультипликатор (см. определение в начале подраздела 4.b) $\chi$ меры $\lambda$ на элементе $g=(a, z)$ принимает значение $a^{k}$, и мера $P_{g}^{\prime}$ имеет плотность

$$
p(x, g)=p\left(\frac{x-z}{a}\right) \frac{1}{a^{k}} .
$$


Легко проверить, что семейство (5.2) $(G-\bar{G})$-инвариантно и что функция $p(\cdot, \cdot)(\mathscr{A} \times \mathscr{B})$-измерима, где $\mathscr{A}$ и $\mathscr{B}$ суть борелевские $\sigma$-алгебры в $\mathscr{X}=\mathbf{R}^{k}$ и $G=\mathbf{R}^{+} \times \mathbf{R}^{k}$ соответственно (в предположении, что $p(\cdot)$ измерима по Борелю). Поэтому, если

$$
I_{m}\left(x_{1}, \ldots, x_{m}\right)=\int\left(\prod_{1}^{m} p\left(x_{i}, g\right)\right) \pi(d g)<\infty
$$

$Q_{m}$-П.в., то по следствию 4.1 формула $\left(4.5^{*}\right)$ с $\pi_{x}^{(m)}(B)$ из $(4.5), \theta=g$, $q\left(x_{i}, \theta\right)=p\left(x_{i}, g\right)$ определяет дезынтеграцию меры $\mu_{m}$ относительно $Q_{m}$, которая $(G-\bar{G})$-инвариантна на $G$-инвариантном множестве $A_{m}$ таком, что $Q_{m}\left(A_{m}^{c}\right)=0$. В рассматриваемом случае $\pi$ есть левоинвариантная мера Хаара на $G=\mathbf{R}^{+} \times \mathbf{R}^{k}$ и может быть записана в виде

$$
\pi(A)=\int_{A} a^{-(k+1)} d a d z_{1} \cdots d z_{k}
$$

(см., например, [12], глава 4, (15.17)).

При $m=1$ для всех $x$ из $\mathscr{X}=\mathbf{R}^{k}$

$$
I_{1}(x)=\int_{0}^{\infty}\left(\int_{\mathbf{R}^{k}} p\left(\frac{x-z}{a}\right) d z\right) a^{-(2 k+1)} d a=\int_{0}^{\infty} a^{-(k+1)} d a=\infty,
$$

так что (5.3) не выполняется для всех $x$. Однако, при выполнении некоторых дополнительных условий можно показать, что (5.3) имеет место $\lambda^{m}$-п.в. (и, следовательно, $Q_{m}$-п.в.) при всех $m \geqslant 2$.

Действительно, предположим, что

$$
p(x) \leqslant K_{1} \quad \text { и } \quad p(x) \leqslant K_{2}|x|^{-r} \quad \text { при }|x| \geqslant 1,
$$

где $K_{1}, K_{2}$ суть некоторые положительные числа и $r>2 k$. Пусть $x_{1}$, $x_{2} \in \mathbf{R}^{k}$ и $x_{1} \neq x_{2}$. Обозначим $x_{12}=\left|x_{1}-x_{2}\right| / 2$ и отметим, что для любого $z$ из $\mathbf{R}^{k}$ и любого $a$ такого, что $0<a \leqslant x_{12}$,

$$
\max \left\{\frac{\left|x_{1}-z\right|}{a}, \frac{\left|x_{2}-z\right|}{a}\right\} \geqslant \frac{x_{12}}{a} \geqslant 1 .
$$

Следовательно, в силу (5.4), для всех $z$ и таких $a$

$$
p\left(\frac{x_{1}-z}{a}\right) p\left(\frac{x_{2}-z}{a}\right) \leqslant K_{2}\left(\frac{a}{x_{12}}\right)^{r}\left(p\left(\frac{x_{1}-z}{a}\right)+p\left(\frac{x_{2}-z}{a}\right)\right) .
$$

Далее, запишем $I_{2}\left(x_{1}, x_{2}\right)$ в виде

$$
I_{2}\left(x_{1}, x_{2}\right)=\int_{0}^{x_{12}} \int_{\mathbf{R}^{k}}+\int_{x_{12}}^{\infty} \int_{\mathbf{R}^{k}}=I_{2}^{\prime}+I_{2}^{\prime \prime},
$$

и отметим, что для любых $a>0$ и $x$ из $\mathbf{R}^{k}$

$$
\int_{\mathbf{R}^{k}} p\left(\frac{x-z}{a}\right) d z=a^{k} .
$$


Вместе с (5.5) это дает $I_{2}^{\prime} \leqslant K_{2} x_{12}^{-r} \int_{0}^{x_{12}} a^{-2 k-1+r} d a<\infty$, при $r>2 k$, и, в силу (5.4),

$$
I_{2}^{\prime \prime} \leqslant \int_{x_{12}}^{\infty} a^{-2 k-1} d a<\infty .
$$

Таким образом, при выполнении условия $(5.4) I_{2}\left(x_{1}, x_{2}\right)<\infty$, если $x_{1} \neq x_{2}$.

Отметим также, что при $k=1$ условие (5.4) легко может быть ослаблено. Действительно, в этом случае

$$
I_{2}\left(x_{1}, x_{2}\right)=\int_{-\infty}^{\infty} p(y) J(y) d y
$$

где при $x_{2}>x_{1}$

$$
J(y)=\int_{0}^{\infty} p\left(y+\frac{x_{2}-x_{1}}{a}\right) \frac{d a}{a^{3}}=\int_{y}^{\infty} p(z) \frac{z-y}{\left(x_{2}-x_{1}\right)^{2}} d z \leqslant \frac{\mathbf{E}|X|+|y|}{\left(x_{2}-x_{1}\right)^{2}}
$$

(и аналогично при $x_{2}<x_{1}$ ). Следовательно, $I_{2}\left(x_{1}, x_{2}\right)<\infty$ при $x_{1} \neq x_{2}$, если $\mathbf{E}|X|<\infty$.

Далее, поскольку, очевидно,

$$
\lambda^{2}\left(x=\left(x_{1}, x_{2}\right): x_{1}, x_{2} \in \mathbf{R}^{k}, x_{1}=x_{2}\right)=0,
$$

то по предложению 4.4 и следствию 4.1 при выполнении условия (5.4) (или лишь при условии $\mathbf{E}|X|<\infty$, если $k=1$ ) соотношение (5.3) имеет место $\lambda^{m}$-п.в. (и $Q_{m}$-п.в.) при всех $m \geqslant 2$.

Предположим теперь, что

$$
Q_{m}\left(A_{m}^{c}\right)=0 \text { при } m \geqslant m_{1},
$$

где $A_{m}$ означает то же, что и в предложении 4.4, с $\mathscr{X}=\mathbf{R}^{k}, \theta=g$ и $q(x, \theta)=p(x, g)$. Тогда в силу следствия 4.1 и замечания 3.5 условие (ii) теоремы 3.1 выполнено с марковским ядром $\widehat{\pi}^{(m)}(\cdot)$, определенным как в следствии 4.1 (с теми же $\mathscr{X}, \theta$ и $q$, что в определении $A_{m}$ в (5.6)). Далее, в рассматриваемом случае справедливость условия (iii) теоремы 3.1 очевидна и условие (i), сформулированное в теореме 2.1, выполнено по замечанию 2.2. Таким образом, при выполнении (5.6) все условия теоремы 3.1 справедливы и поэтому верно ее заключение.

Резюмируя сказанное выше, мы видим, что если $\mathbf{E}|X|<\infty$ при $k=1$, и если выполнено условие (5.4) при $k>1$, то существуют версии апостериорных вероятностей $\hat{\pi}_{.}^{(m)}(\cdot), m \geqslant 2$, определенные формулой $\left(4.5^{*}\right)$ с $\mathscr{X}=\mathbf{R}^{k}, \theta=g, q\left(x_{i}, \theta\right)=p\left(x_{i}, g\right)$, для которых

$$
P_{g}\left(x: \hat{\pi}_{(x)_{m}}^{(m)} \Rightarrow \delta_{g}\right)=1 \quad \text { при всех } g \in G \text {. }
$$

Авторы выражают свою благодарность А. Беллоу и Л. Ле Каму за помощь в поиске известных результатов по лифтингу. 


\section{СПИСОК ЛИТЕРАТУРЫ}

1. Brieman L., Le Cam L., Schwartz L. Consistent estimates and zero-one set. - Ann. Math. Statist., 1964, v. 35, p. 157-161.

2. Chatterji $S . D$. Les martingales et leurs applications analytiques. - In Ecole d'Été de Probabilites: Processus Stochastiques, (Ed. by Badrikian A., Hennequin P.L.), Lecture Notes in Math., 1973, v. 307, p. 27-164.

3. Dellacherie C., Meyer P-A. Probabilities and Potential, Part B. Amsterdam: NorthHolland, 1982, $454 \mathrm{p}$.

4. Diaconis P., Freedman D.A. On the consistency of Bayes estimates (with discussion). - Ann. Statist., 1986, v. 14, p. 1-26.

5. Doob J. L. Applications of the theory of martingales. - Coll. Int. du CNRS, Paris, 1949, № 13, p. 23-27.

6. Doob J. L. Measure Theory. New York: Springer-Verlag, 1994.

7. Dudley R.M. Real Analysis and Probability. Pacific Grove: Wadsworth\&Brooks/Cole, 1989.

8. Eaton M. L. Multivariate Statistics: a Vector Space Approach. New York: Wiley, $1983,512 \mathrm{p}$.

9. Ferguson T.S. Location and scale parameters in exponential families of distributions. - Ann. Math. Statist., 1962, v. 33, p. 986-1001.

10. Freedman D.A. On the asymptotic behavior of Bayes estimates in the discrete case I. - Ann. Math. Statist., 1963, v. 34, p. 1386-1403.

11. Freedman D.A. On the asymptotic behavior of Bayes estimates in the discrete case II. - Ann. Math. Statist., 1965, v. 36, p. 454-456.

12. Хьюитm Э. Абстрактный гармонический анализ. Т. 1. М.: Наука, 1975, 654 с.

13. Ionescu Tulcea A., Ionescu Tulcea $C$. On the existence of lifting commuting with left translations of an arbitrary locally compact group. - In Proc. Fifth Berkeley Symp. Math. Statist. Probab., 1967, v. 2, p. 63-97.

14. Johnson R.A. Strong liftings commuting with minimal distal flows. - Pacific J. Math., 1980 , v. 90 , p. $77-85$.

15. Куратовский К. Топология. Т. 1. М.: Мир, 1966, 594 с.

16. Le Cam L. On some asymptotic properties of maximum likelihoogd estimates and related Bayes estimates. - In Univ. California Publ. Statist. Univ. of California Press, Berkeley, 1953, v. 1, p. 277-330.

17. Le Cam L. Limits of experiments. - In Proc. 6th Berkeley Symp. Math. Statist. Probab., Univ. of California Press, Berkeley, 1972, v. 1, p. 245-261.

18. Le Cam L. Asymptotic Methods in Statistical Decision Theory. New York: SpringerVerlag, 1986, $742 \mathrm{p}$.

19. Леман Э. Проверка статистических гипотез. М.: Наука, 1979, 408 с.

20. Lo A. Y. Consistency in the location model: the undominated case. - Ann. Statist., 1984 , v. 12 , p. $1584-1587$.

21. Lo A. Y., Cabrera J. Bayes procedures for rotationally symmetric models on the sphere. - Ann. Statist., 1987, v. 15, p. 1257-1268.

22. Невё Ж. Математические основы теории вероятностей. М.: Мир, 1969, 309 c.

23. Parthasarathy K. R. Probability Measures on Metric Spaces. New York: Academic Press, 1967, $276 \mathrm{p}$.

24. Royden H. L. Real Analysis. New York: Macmillan, 1988, 444 p.

25. Schwartz L. On Bayes procedures. - Z. Wahrscheinlichkeitstheor. verw. Geb., 1965, B. 4 , S. $10-26$.

26. Watson J. S. Statistics on the Spheres. New York: Wiley, 1983.

Поступила в редакцию 12.III. 2001 